\title{
On the $\mathrm{SU}(2 \mid 1) \mathrm{WZNW}$ model and its statistical mechanics applications
}

\author{
Hubert Saleur* and Volker Schomerus** \\ *Service de Physique Théorique, CEA Saclay, \\ F-91191 Gif-sur-Yvette, France \\ **DESY Theory Group, DESY Hamburg \\ Notkestrasse 85, D-22603 Hamburg, Germany \\ *Physics Department \\ University of Southern California \\ Los Angeles CA 90089-0484, USA
}

Nov. 2006

\begin{abstract}
Motivated by a careful analysis of the Laplacian on the supergroup $\mathrm{SU}(2 \mid 1)$ we formulate a proposal for the state space of the $\mathrm{SU}(2 \mid 1)$ WZNW model. We then use properties of $\widehat{\mathrm{sl}}(2 \mid 1)$ characters to compute the partition function of the theory. In the special case of level $k=1$ the latter is found to agree with the properly regularized partition function for the continuum limit of the integrable $\operatorname{sl}(2 \mid 1) 3-\overline{3}$ super-spin chain. Some general conclusions applicable to other WZNW models (in particular the case $k=-1 / 2$ ) are also drawn.
\end{abstract}

e-mail:hubert.saleur@cea.fr, volker.schomerus@desy.de 


\section{Introduction}

The SU(2|1) WZNW model is a key example of the sigma models with supergroup targets that appear in the supersymmetric description of non interacting disordered systems in low dimensional statistical mechanics. The first occurrence of this model probably arose via a supersymmetrization of the path integral for two copies of the two dimensional critical Ising model. It was shown in [1] how a $\beta \gamma$ system (with central charge $c=-1$ ) could be introduced to cancel out the pair of free Majorana fermions (regrouped for convenience into a Dirac fermion) path integrals

$$
Z=\int\left[d \psi d \psi^{\dagger} d \beta d \gamma\right] \exp \left[S_{0}+\delta S\right]=1
$$

where

$$
S_{0}=\int \frac{d^{2} x}{2 \pi}\left(\psi^{\dagger} \bar{\partial} \psi+\bar{\psi}^{\dagger} \partial \bar{\psi}+\beta \bar{\partial} \gamma+\bar{\beta} \partial \bar{\gamma}\right)
$$

and

$$
\delta S=\int \frac{d^{2} x}{2 \pi} i \frac{m(x)}{2}\left(\bar{\psi}^{\dagger} \psi-\psi^{\dagger} \bar{\psi}+\bar{\beta} \gamma-\beta \bar{\gamma}\right)
$$

The theory without random mass $m(x)=0$ is obviously a free $\operatorname{OSP}(2 \mid 2)$ theory, which can be considered as a $\mathrm{SU}(2 \mid 1) \mathrm{WZNW}$ model at level $k=-1 / 21$. Averaging over disorder produces a marginally irrelevant current current perturbation of this WZNW model. This is crucial to understanding the (logarithmic) corrections to pure Ising model scaling. The deep infrared (IR) behavior however is not changed by the disorder, which corresponds to the fact that (1.2) is a simple free theory, with pure fermionic correlators identical to those of the usual Ising model.

The second occurrence of the $\mathrm{SU}(2 \mid 1)$ model is more involved. It arises in the study of $2+1$ dimensional spin-full electrons in the presence of a random (non abelian) gauge potential. The supersymmetrization of the path integral for two copies of the Dirac fermions produces a free $\operatorname{OSP}(4 \mid 4)$ theory which has been argued to flow to the $\mathrm{SU}(2 \mid 1)$ WZNW model at level one under the action of the disorder [2]. The nature of the spectrum and correlation functions play an important role in the description of the electronic wave functions at that fixed point.

\footnotetext{
${ }^{1}$ Our conventions are such that the sub SU(2) algebra has level $k$. In part of the literature, the level is defined as $-2 \times$ ours, so the free system in (1.1) has $k=1$ there.
} 
Previous works on the $\mathrm{SU}(2 \mid 1)$ model have focused on some correlation functions [3], 4] and on the construction of some characters [5], but a complete picture of the theory has been missing.

Indeed, the analysis of WZNW on supergroups is notoriously difficult, even for the simplest case of GL(1|1) [6]. In a recent paper [7], we have shown how a careful study of the particle limit (in particular, of the simultaneous left and right invariant actions on the space of functions on the group) could provide considerable insight into this problem. Combining this insight with some additional input from the representation theory of the current algebras allowed us to formulate a complete proposal for the state space of the theory in the case of GL(1|1). The latter involves a rather intricate mixing of left and right movers that is intimately related to the representation theory of Lie superalgebras, in particular to the importance of indecomposable representations. We were then able to check this proposal through an exact construction of the theory in the continuum formulation.

The aim of this work is to extend the lessons we have learned in [7] to a non-abelian setup, using $\mathrm{SU}(2 \mid 1)$ as the simplest non-trivial example 2 Once more, the analysis of the particle limit (section 2) along with some input from the representation theory of the $\operatorname{sl}(2 \mid 1)$ current algebra (section 3) shall provide all the necessary ingredients for the construction of the field theory state space (section 4), in close analogy to our previous investigation of the GL(1|1) model. In the present case we shall not attempt to verify the structure of the state space through calculations of correlators, though this would be possible as well (see [8]). Instead we shall use results on an integrable sl(2|1) spin chain to test our continuum constructions. Such a spin chain was first investigated in [9] as a discrete version of the $\mathrm{SU}(2 \mid 1)$ WZNW model. We shall see that both approaches are consistent. The comparison, however, is a bit subtle, mainly due to the fact that the supergroup $\mathrm{SU}(2 \mid 1)$ has an indefinite metric. While this poses no problem for the (algebraic) conformal field theory analysis, the computation of the partition function on the lattice suffers from divergencies which need to be regularized. We shall do this through some appropriate analytic continuation. In this sense, our analysis also supports a particular prescription for extracting information from spin chains with an indefinite metric.

\footnotetext{
${ }^{2}$ To be more precise, we shall consider the universal cover of $\mathrm{SU}(2 \mid 1)$ in which the abelian, time-like circle is replaced by the real line. We shall comment on this in much more detail in section 4 .
} 


\section{The minisuperspace analysis}

The aim of this section is to decompose the space of functions on the supergroup $\mathrm{SU}(2 \mid 1)$ into (generalized) eigenfunctions of the quadratic Casimir element in the regular representations. Since the Casimir commutes with the generator, the eigenspaces may be decomposed into representation of the Lie superalgebra $\mathrm{sl}(2 \mid 1)$. It is therefore useful to have some background on the representation theory of $\operatorname{sl}(2 \mid 1)$. We shall review a few known facts below before addressing the harmonic analysis. More details can be found e.g. in [10, 11].

\subsection{The Lie superalgebra $\operatorname{sl}(2 \mid 1)$}

In this subsection we provide a short overview on finite dimensional representations of $\operatorname{sl}(2 \mid 1)$. Rather than reproducing a complete list of such representations we shall focus on those that are relevant below, namely on Kac modules and the projective covers of atypicals.

\subsubsection{The defining relations of $\operatorname{sl}(2 \mid 1)$}

The even part $\mathfrak{g}^{(0)}=\operatorname{gl}(1) \oplus \operatorname{sl}(2)$ of the Lie superalgebra $\mathfrak{g}=\operatorname{sl}(2 \mid 1)$ is generated by four bosonic elements $H, E^{ \pm}$and $B$ which obey the commutation relations

$$
\left[H, E^{ \pm}\right]= \pm E^{ \pm} \quad, \quad\left[E^{+}, E^{-}\right]=2 H \quad, \quad\left[B, E^{ \pm}\right]=[B, H]=0
$$

In addition, there exist two fermionic multiplets $\left(F^{+}, F^{-}\right)$and $\left(\bar{F}^{+}, \bar{F}^{-}\right)$which generate the odd part $\mathfrak{g}^{(1)}$. They transform as $\left( \pm \frac{1}{2}, \frac{1}{2}\right)$ with respect to the even subalgebra, i.e.

$$
\begin{aligned}
{\left[H, F^{ \pm}\right] } & = \pm \frac{1}{2} F^{ \pm} & {\left[H, \bar{F}^{ \pm}\right] } & = \pm \frac{1}{2} \bar{F}^{ \pm} \\
{\left[E^{ \pm}, F^{ \pm}\right] } & =\left[E^{ \pm}, \bar{F}^{ \pm}\right]=0 & {\left[E^{ \pm}, F^{\mp}\right] } & =-F^{ \pm} \\
{\left[B, F^{ \pm}\right] } & =\frac{1}{2} F^{ \pm} & {\left[B, \bar{F}^{ \pm}\right] } & =-\frac{1}{2} \bar{F}^{ \pm} .
\end{aligned}
$$

Finally, the fermionic elements possess the following simple anti-commutation relations

$$
\left\{F^{ \pm}, F^{\mp}\right\}=\left\{\bar{F}^{ \pm}, \bar{F}^{\mp}\right\}=0 \quad\left\{F^{ \pm}, \bar{F}^{ \pm}\right\}=E^{ \pm} \quad\left\{F^{ \pm}, \bar{F}^{\mp}\right\}=B \mp H
$$

among each other. Formulas (2.1) to (2.3) provide a complete list of relations in the Lie superalgebra $\operatorname{sl}(2 \mid 1)$. 


\subsubsection{Kac modules and irreducible representations}

Kac modules [12] are the basic tool in the construction of irreducible representations. In the case of $\mathfrak{g}=\mathrm{sl}(2 \mid 1)$, these form a 2-parameter family $\{b, j\}$ of $8 j$-dimensional representations. We may induce them from the $2 j$-dimensional representations $\left(b-\frac{1}{2}, j-\frac{1}{2}\right)$ of the bosonic subalgebra $\mathfrak{g}^{(0)}$ by applying the pair $F^{ \pm}$of fermionic elements. Our label $b \in \mathbb{C}$ denotes a gl(1)-charge and spins of $\operatorname{sl}(2)$ are labeled by $j=\frac{1}{2}, 1, \ldots$ The dual construction which promotes the fermionic generators $\bar{F}^{ \pm}$to creation operators, yields anti-Kac modules $\{\overline{b, j}\}$ ( $b$ and $j$ take the same values as above). The bosonic content of (anti-)Kac modules may be read off rather easily from their construction,

$$
\left.\left.\{b, j\}\right|_{\mathfrak{g}^{(0)}} \cong\{\overline{b, j}\}\right|_{\mathfrak{g}^{(0)}} \cong\left(b-\frac{1}{2}, j-\frac{1}{2}\right) \oplus(b, j) \oplus(b, j-1) \oplus\left(b+\frac{1}{2}, j-\frac{1}{2}\right) .
$$

For generic values of $b$ and $j$, the modules $\{b, j\}$ and $\{\overline{b, j}\}$ are irreducible and isomorphic. At the points $\pm b=j$, however, they degenerate, i.e. the representations are indecomposable and no longer isomorphic. In fact, Kac and anti-Kac modules are then easily seen to possess different invariant subspaces. To be more precise the (anti-)Kac modules $\{ \pm j, j\}$ and $\{\overline{ \pm j, j}\}$ are built from two atypical representations such that

$$
\begin{array}{ll}
\{ \pm j, j\}: & \{j\}_{ \pm} \longrightarrow\left\{j-\frac{1}{2}\right\}_{ \pm} \\
\{\overline{ \pm j, j}\}: & \left\{j-\frac{1}{2}\right\}_{ \pm} \longrightarrow\{j\}_{ \pm} .
\end{array}
$$

The atypical irreducible representations $\{j\}_{ \pm}$that appear in these small diagrams are $4 j+1$ dimensional. With respect to the even subalgebra they decompose according to

$$
\left.\{j\}_{ \pm}\right|_{\mathfrak{g}^{(0)}}= \begin{cases}(j, j) \oplus\left(j+\frac{1}{2}, j-\frac{1}{2}\right) & , \text { for }+ \text { and } j=\frac{1}{2}, 1, \ldots \\ (-j, j) \oplus\left(-\left(j+\frac{1}{2}\right), j-\frac{1}{2}\right) & , \text { for }- \text { and } j=\frac{1}{2}, 1, \ldots\end{cases}
$$

For $j=0$, only the trivial representation (0) occurs. It is also useful to introduce the characters of these representations. By definition, these are obtained as

$$
\chi_{\mathcal{R}}(z, \xi)=\operatorname{str}_{\mathcal{R}}\left(\xi^{B} z^{H}\right)
$$

where the super-trace extends over all states in the representation $\mathcal{R}$ of $\operatorname{sl}(2 \mid 1)$. For Kac modules the character is rather simple. In fact, it factorizes

$$
\chi_{\{b, j\}}(\xi, z)=\xi^{b-1 / 2} \chi_{f}(\xi, z) \sum_{l=-j+1 / 2}^{l=j-1 / 2} z^{l}
$$


with a fermionic contribution $\chi_{f}$ that is independent of the Kac module under consideration,

$$
\chi_{f}(\xi, z)=1-\xi^{1 / 2} z^{1 / 2}-\xi^{1 / 2} z^{-1 / 2}+\xi .
$$

The characters of atypical representations can be obtained easily form their decomposition formulas (2.6). We would like to pursue a rather different route here that uses the decomposition (2.5) of Kac modules into atypicals. The first formula implies that

$$
\chi_{\{ \pm j, j\}}(\xi, z)=\chi_{\{j\}_{ \pm}}(\xi, z)-\chi_{\{j-1 / 2\}_{ \pm}}(\xi, z) .
$$

We can solve these equations for the characters of atypical representations by the following infinite sums

$$
\chi_{\{j-1 / 2\}_{ \pm}}(\xi, z)=-\sum_{n=0}^{\infty} \chi_{\{ \pm j \pm n / 2, j+n / 2\}}(\xi, z)
$$

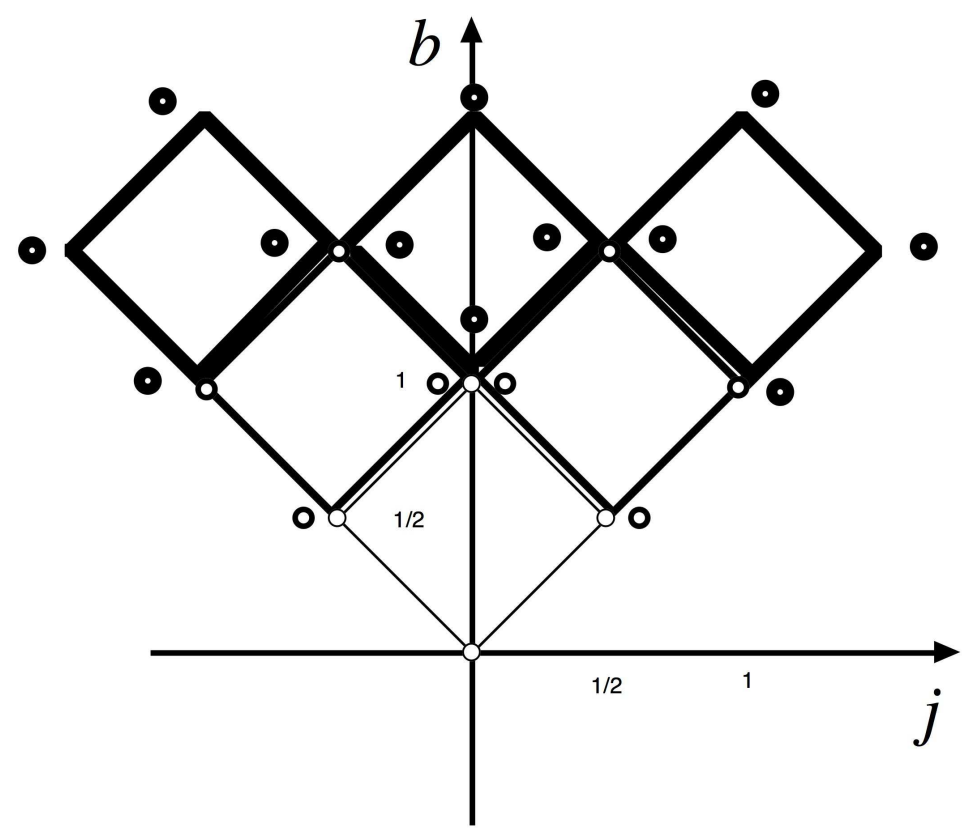

Figure 1: A graphical illustration of how characters for an atypical representation can be obtained as an infinite sum of characters of Kac modules. Here the one dimensional atypical identity 0 appears as a sum over $\{1 / 2,1 / 2\}$ (thin lines and dots), $\{1,1\}$ (medium lines and dots), $\{3 / 2,3 / 2\}$ (thick lines and dots) etc. All spurious contributions (that is, the whole tower but the origin) appear twice, and they disappear by cancellations of bosonic and fermionic degrees of freedom. The diagram corresponds to the choice of plus sign in formula (2.8). 
One may check by explicit computation that the contributions from all but two bosonic multiplets cancel each other in the infinite sum through a mechanism that is visualized in Figure 1. The remaining two terms certainly agree with the decomposition formulas (2.6). Our derivation here may seem like a rather complicated path for such a simple result, but we shall see later that the same trick works for characters of atypical affine representations which are otherwise difficult to obtain.

\subsubsection{Projective covers of atypical irreducible modules}

By definition, the projective cover of a representation $\{j\}_{ \pm}$is the largest indecomposable representation $\mathcal{P}^{ \pm}(j)$ which has $\{j\}_{ \pm}$as a subrepresentation (its socle). We do not want to construct these representations explicitly here. Instead, we shall display how they are composed from atypicals. The projective cover of the trivial representation is an 8-dimensional module of the form

$$
\mathcal{P}(0): \quad\{0\} \longrightarrow\left\{\frac{1}{2}\right\}_{+} \oplus\left\{\frac{1}{2}\right\}_{-} \longrightarrow\{0\}
$$

For the other atypical representations $\{j\}_{ \pm}$with $j=\frac{1}{2}, 1, \ldots$ one finds the following diagram,

$$
\mathcal{P}^{ \pm}(j): \quad\{j\}_{ \pm} \longrightarrow\left\{j+\frac{1}{2}\right\}_{ \pm} \oplus\left\{j-\frac{1}{2}\right\}_{ \pm} \longrightarrow\{j\}_{ \pm} .
$$

These representation spaces are $16 j+4$-dimensional. Let us agree to absorb the superscript \pm on $\mathcal{P}$ into the argument, i.e. $\mathcal{P}^{ \pm}(j)=\mathcal{P}( \pm j)$, wherever this is convenient.

\subsection{Functions on the supergroup $\mathrm{SU}(2 \mid 1)$}

Now we are prepared to analyze the space of functions on the supergroup $\mathrm{SU}(2 \mid 1)$. For this purpose, let us introduce coordinates through the following explicit decomposition of elements $U \in \mathrm{SU}(2 \mid 1)$,

$$
U=e^{i \bar{\eta}_{\nu} \bar{F}^{\nu}} e^{i z B} g e^{i \eta_{\nu} F^{\nu}}
$$

Here, the bosonic base $\mathrm{SU}(2) \times \mathbb{R}$ is parametrized by an element $g \in \mathrm{SU}(2) \cong \mathrm{S}^{3}$ along with the time-like variable $z$. In these coordinates, the generators of the right regular 
action read

$$
\begin{gathered}
R_{F^{ \pm}}=-i \partial_{ \pm} \quad, \quad R_{E^{ \pm}}=R_{E^{ \pm}}^{0}+\eta_{\mp} \partial_{ \pm} \\
R_{H}=R_{H}^{0}+\frac{1}{2} \eta_{-} \partial_{-}-\frac{1}{2} \eta_{+} \partial_{+} \quad, \quad R_{B}=-i \partial_{z}-\frac{1}{2} \eta_{-} \partial_{-}-\frac{1}{2} \eta_{+} \partial_{+} \\
R_{\bar{F}^{ \pm}}=i e^{-i z / 2} D_{\nu( \pm 1 / 2)}^{1 / 2}(g) \bar{\partial}_{-\nu}+i \eta_{ \pm}\left(R_{E^{ \pm}}^{0}+\eta_{\mp} \partial_{ \pm}\right)-i \eta_{\mp}\left(i \partial_{z} \mp R_{H}^{0}\right)
\end{gathered}
$$

where $R_{X}^{0}$ are the generators of the right regular representation of $\mathrm{SU}(2)$. They act on the matrix elements $D_{a b}^{j}(g), a, b=-j,-j+1, \ldots j$, according to

$$
\begin{gathered}
R_{H}^{0} D_{a b}^{j}(g)=b D_{a b}^{j}(g) \quad, \quad R_{E^{+}}^{0} D_{a b}^{j}(g)=\sqrt{(j+b+1)(j-b)} D_{a(b+1)}^{j}(g), \\
R_{E^{-}}^{0} D_{a(b+1)}^{j}(g)=\sqrt{(j+b+1)(j-b)} D_{a b}^{j}(g) .
\end{gathered}
$$

Matrix elements with $j=1 / 2$ appear as coefficients in the differential operators $R_{\bar{F}^{ \pm}}$ and their behavior under the action of $R_{X}^{0}$ plays an important role in checking that the above generators of the right regular representation obey the defining relations of $\operatorname{sl}(2 \mid 1)$. Formulas for the left regular representation may be obtained similarly,

$$
\begin{gathered}
L_{\bar{F}^{ \pm}}=-i \bar{\partial}_{ \pm} \quad, \quad L_{E^{ \pm}}=L_{E^{ \pm}}^{0}-\bar{\eta}_{\mp} \bar{\partial}_{ \pm} \\
L_{H}=L_{H}^{0}+\frac{1}{2} \bar{\eta}_{-} \bar{\partial}_{-}-\frac{1}{2} \bar{\eta}_{+} \bar{\partial}_{+} \quad, \quad L_{B}=i \partial_{z}+\frac{1}{2} \bar{\eta}_{-} \bar{\partial}_{-}+\frac{1}{2} \bar{\eta}_{+} \bar{\partial}_{+}, \\
L_{F^{ \pm}}=i e^{-i z / 2} D_{( \pm 1 / 2) \nu}^{j}(g) \partial_{-\nu}+i \bar{\eta}_{ \pm}\left(L_{E^{ \pm}}^{0}-\bar{\eta}_{\mp} \bar{\partial}_{ \pm}\right)+i \bar{\eta}_{\mp}\left(i \partial_{z} \mp L_{H}^{0}\right)
\end{gathered}
$$

It is probably not necessary to stress that left and right generators (anti-)commute with respect to each other.

By construction (see however [8]), the generators of the left and right regular representation act on the space of all Grassmann valued functions with square integrable coefficients on the bosonic base, i.e. on the space

$$
L_{2}(\mathrm{SU}(2 \mid 1)):=L_{2}(S U(2) \times \mathbb{R}) \otimes \Lambda\left(\eta_{ \pm}, \bar{\eta}_{ \pm}\right)
$$

where $\Lambda\left(\eta_{ \pm}, \bar{\eta}_{ \pm}\right)$denotes the Grassmann algebra that is generated by our four fermionic coordinates $\eta_{ \pm}$and $\bar{\eta}_{ \pm}$. With respect to the left regular action, the space of square integrable functions can be shown to decompose as follows,

$$
\begin{aligned}
L_{2}(\mathrm{SU}(2 \mid 1)) \cong_{L} \sum_{j=1 / 2}^{\infty} \sum_{b \neq \pm j} 4 j\left(\{-b, j\} \oplus\{b, j\}^{\prime}\right) \oplus \\
\quad \oplus \sum_{j}(2 j+1)\left(\mathcal{P}_{j}^{+} \oplus \mathcal{P}_{j}^{-}\right) \oplus 2 j\left(\mathcal{P}_{j}^{+} \oplus \mathcal{P}_{j}^{-}\right)^{\prime} .
\end{aligned}
$$


Here, the summation runs over $j=0,1 / 2,1, \ldots,\{b, j\}$ denotes the typical representations of $\operatorname{sl}(2 \mid 1)$ and $\mathcal{P}_{j}^{ \pm}$are the projective covers of the atypical representations $\{j\}_{ \pm}$. Most of our conventions can be found e.g. in [10]. A prime' on a representation means that the degree is inverted, i.e. that fermionic vectors become bosonic and vice versa. The result is a special case of the general observation made in [13] and it generalizes a similar decomposition we described in [7] for the left regular action of gl(1|1). The interested reader can find an explicit proof in Appendix A. Let us comment that the decomposition of the left regular action displays the same violation of the Peter-Weyl theorem as in the case of GL(1|1). In particular, since the quadratic Casimir is not diagonalizable in the projective covers $\mathcal{P}_{j}^{ \pm}$, the Laplacian on the supergroup $\mathrm{SU}(2 \mid 1)$ can only be brought into Jordan normal form. The blocks can reach a rank up to three.

The functions on our supergroup carry another (anti-)commuting action of the Lie superalgebra $\mathfrak{g}$ by left derivations. There is a corresponding decomposition which is certainly identical to the decomposition above. A more interesting problem is to decompose the space of functions with respect to the graded product $\mathfrak{g} \otimes \mathfrak{g}$ in which the first factor acts through the left regular action while for the second factor we use the right regular action. In the typical sector, the $4 j \mid 4 j$-dimensional multiplicity spaces in the first line of eq. (2.17) get promoted to typical representations of the right regular action, i.e.

$$
L_{2}(\mathrm{SU}(2 \mid 1)) \cong_{L-R} \sum_{j=1 / 2}^{\infty} \sum_{b \neq \pm j}\left(\{b, j\}_{L} \otimes\{-b, j\}_{R}\right) \oplus \mathcal{J}
$$

where $\mathcal{J}$ is a single indecomposable, containing all the atypical building blocks. Its structure may be summarized by the following picture

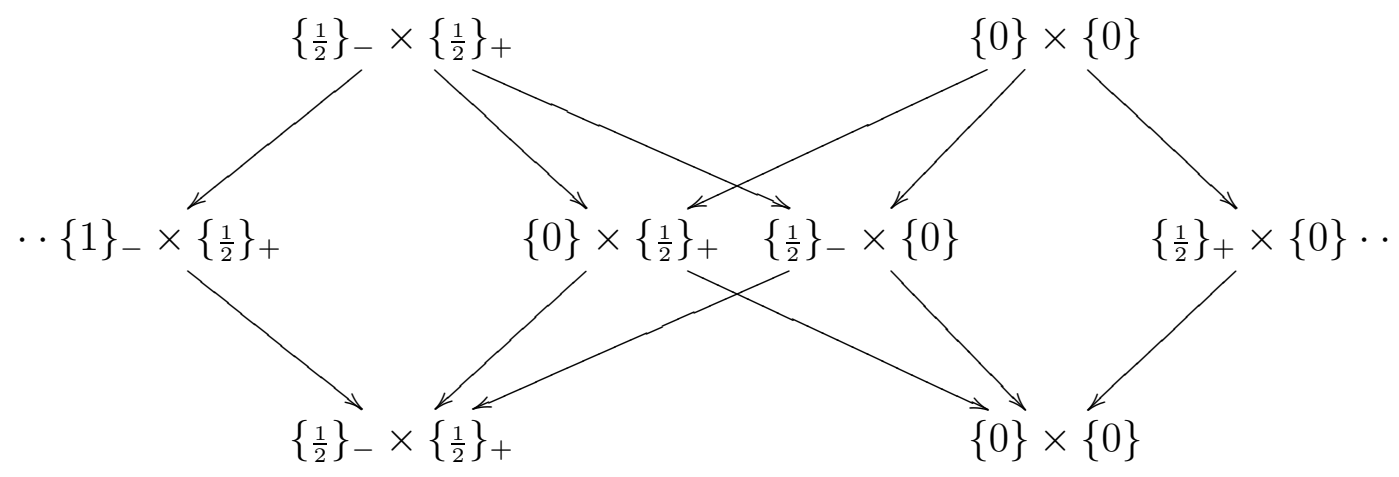

This diagram is the natural extension of the corresponding picture for GL(1|1). It extends to infinity in both directions and combines all the atypical sectors into a single 
indecomposable representation. Note that by construction, each projective cover in the decomposition of the right regular representation appears with the correct multiplicity. We shall see below how this picture is modified in the full quantum theory.

\section{Representation theory of the affine algebra}

The previous analysis of the particle limit applies to all sigma models on $\mathrm{SU}(2 \mid 1)$, but the information it provides is usually not sufficient in order to reconstruct the entire field theory from it. This is very different for the WZNW model in which the entire spectrum can be generated from particle wave functions through current algebra symmetries. We need some facts on the representation theory of the $\operatorname{sl}(2 \mid 1)$ current algebra and shall provide them in the following section. All the results we collect here are well known from [14, 5, 15, 16, 17]. Their derivation, however, is somewhat original. In particular, we shall use a simple, but highly efficient prescription to construct characters of atypical representations of $\widehat{\mathrm{sl}}(2 \mid 1)$ through infinite sums over typicals. This extends the formula (2.8) we have discussed in section 2 to an infinite dimensional setting, thereby generalizing a trick that has first been proposed in the context of the gl(1|1) current algebra [18].

\subsection{Some basic ingredients}

Irreducible representations of the affine $\mathrm{sl}(2 \mid 1)$ algebra can be built over the irreducible typical representations $\{b, j\}$ with $j=1 / 2, \ldots, k / 2$ as well as over the atypicals $\{j\}_{ \pm}$ with $j=0,1 / 2,1, \ldots, k / 2$. Ground states in the former set of representations possess conformal dimension

$$
h_{\{b, j\}}=\left(j^{2}-b^{2}\right) /(k+1)
$$

while the conformal dimension for ground states in the latter set vanishes. Following the work [14] of Bowcock et al. we shall divide these representations into three different classes. The generic class I representations occur for $\{b, j\}$ with $b \neq j_{m}^{ \pm}$where we defined

$$
j_{m}^{ \pm}:= \pm j+m(k+1) \text { for } m \text { integer } .
$$

Class II representations include those erected over $\{b, j\}$ with $b=j_{m}^{ \pm}, m \neq 0$, along with the sectors generated from atypicals $\{j\}_{ \pm}, j \neq 0$. The vacuum representation that is generated from the atypical $\{0\}$ is the only member of the final class, which we denote as class 
IV for historical reasons. Our aim is to describe the singular vectors in the corresponding Verma modules and to provide the associated formulas for the super-characters

$$
\chi_{\mathcal{R}}(q, z, \xi):=\operatorname{str}_{\mathcal{R}}\left(q^{L_{0}-\frac{c}{24}} \xi^{B_{0}} z^{H_{0}}\right)
$$

of irreducible representations. The results we describe have first appeared in [14].

Before we start our discussion of characters let us quickly recall that it is possible to construct $\mathrm{sl}(2 \mid 1)$ currents in terms of decoupled bosonic and fermionic variables. To be more precise, we introduce a set of bosonic currents $e^{ \pm}(z), h(z), b(z)$ and assume them to satisfy the operator product expansions of an affine $\operatorname{sl}(2)$ algebra at level $k-1$. In addition, let us introduce two sets of fermionic fields $p^{a}$ and $\theta^{a}$ obeying the canonical relations

$$
\theta^{a}\left(z_{1}\right) p^{b}\left(z_{2}\right) \sim \frac{\delta_{a b}}{z_{1}-z_{2}}+\ldots
$$

Then we can construct an $\operatorname{sl}(2 \mid 1)$ current algebra at level $k$ through the following prescription,

$$
\begin{aligned}
& E^{+}(z)=e^{+}(z)+: \theta^{1} p^{2}:(z), \quad H(z)=h(z)+\frac{1}{2}:\left(\theta^{1} p^{1}-\theta^{2} p^{2}\right):(z), \\
& E^{-}(z)=e^{-}(z)+: \theta^{1} p^{2}:(z), \quad B(z)=b(z)-\frac{1}{2}:\left(\theta^{1} p^{1}+\theta^{2} p^{2}\right):(z), \\
& F^{+}(z)=p^{2}(z) \quad, \quad \bar{F}^{+}=\theta^{2} e^{+}(z)+\theta^{1}(b+h)(z)-: \theta^{1} \theta^{2} p^{2}:(z), \\
& F^{-}(z)=p^{1}(z) \quad, \quad \bar{F}^{-}=\theta^{1} e^{-}(z)+\theta^{2}(b-h)(z)+: \theta^{1} \theta^{2} p^{2}:(z) .
\end{aligned}
$$

Since the fermionic fields $\theta^{a}$ and $p^{a}$ are supposed to commute with the bosonic fields $e^{ \pm}(z), h(z)$ and $b(z)$, the characters of typical representations factorize with the factors $\theta_{1}(y, q)$ arising from the fermionic pairs. The shift $j \rightarrow j-1 / 2$ in the bosonic contribution may be traced back to a similar shift in the labeling of typical $\mathrm{sl}(2 \mid 1)$ representations, see eq. (2.4).

\subsection{Typical (class I) representations}

The generic class I representations have no singular vectors except from the ones that arise through the representations of a bosonic $\mathrm{su}(2)$ current algebra at level $k-1$. In this sense, they may be considered the typical representations of the affine sl(2|1) algebra. 
The statement implies a precise expression for the characters of class I representations

$$
\begin{gathered}
\chi_{\{b, j\}}^{I}(q, z, \xi)=\frac{q^{-b^{2} /(k+1)}}{\xi^{-b} \eta^{3}(q)} \theta_{1}\left(z^{1 / 2} \xi^{1 / 2}, q\right) \theta_{1}\left(z^{-1 / 2} \xi^{1 / 2}, q\right) \chi_{j-1 / 2}^{k-1}(z, q) \\
\text { where } \theta_{1}(y, q)=-i y^{1 / 2} q^{1 / 8} \prod_{n=1}^{\infty}\left(1-q^{n}\right)\left(1-y q^{n}\right)\left(1-y^{-1} q^{n-1}\right)
\end{gathered}
$$

and $b \neq j_{m}^{ \pm}$and $1 / 2 \leq j \leq k / 2$. We also recall that the $\mathrm{su}(2)$ characters are given by

$$
\chi_{j-1 / 2}^{k-1}(z, q)=q^{\frac{j^{2}}{k+1}-\frac{1}{8}} z^{j-1} \frac{\sum_{a} q^{(k+1) a^{2}+2 a j}\left(z^{a(k+1)}-z^{-a(k+1)-2 j}\right)}{\prod_{n=1}^{\infty}\left(1-z q^{n}\right)\left(1-z^{-1} q^{n-1}\right)\left(1-q^{n}\right)} .
$$

We shall use the symbol $\{b, j\}^{\wedge}$ for these irreducible representations of the affine algebra. The formulas are easy to understand: they follow directly from the representation (3.1) of the $\operatorname{sl}(2 \mid 1)$ current algebra. In fact, each pair of fermionic fields contributes a factor $\theta_{1} / \eta$ while the bosonic $\mathrm{sl}(2)$ and $\mathrm{u}(1)$ current algebras are responsible for the characters $\chi^{k-1}$ and an additional factor $\eta^{-1}$, respectively.

\subsection{Atypical (class II) representations}

Nothing prevents us from evaluating the previous character formulas at the points $b=j_{m}^{ \pm}$. But the resulting functions turn out to be the characters of indecomposable representations $\left\{j_{m}^{ \pm}, j\right\}^{\wedge}$ which contain one fermionic singular multiplet. In order to state this more precisely, let us consider in more detail the set of atypical labels,

$$
A:=\left\{\left\{j_{m}^{ \pm}, j\right\} \mid 1 / 2 \leq j \leq k / 2 ; m \in \mathbb{Z}\right\}
$$

The set $A$ is visualized in Figure 2. Our picture shows clearly that the projection to the $b$-coordinate of each element in $A$ is injective and hence it can be used to enumerate our atypical labels. Note, however, that values $b \in(k+1) / 2 \mathbb{Z}$ are omitted. This motivates to introduce an improved enumeration map $\hat{\nu}$ from $A$ to non-zero half-integers which is defined by

$$
\begin{array}{ll}
\hat{\nu}\left(\left\{j_{m}^{+}, j\right\}\right)=j_{m}^{+}-m \text { for } m \geq 0 \quad, \quad \hat{\nu}\left(\left\{j_{m}^{-}, j\right\}\right)=j_{m}^{-}-m+1 / 2 \text { for } m>0 \\
\hat{\nu}\left(\left\{j_{m}^{-}, j\right\}\right)=j_{m}^{-}+m \text { for } m \leq 0 \quad, \quad \hat{\nu}\left(\left\{j_{m}^{+}, j\right\}\right)=j_{m}^{+}+m-1 / 2 \text { for } m<0 .
\end{array}
$$

By construction, $\hat{\nu}$ is not only an injection but its image now also consists of all nonzero half-integers. We may view $\hat{\nu}$ as an affine version of the enumeration map $\nu(\{ \pm j, j\})= \pm j$ for representations of $\operatorname{sl}(2 \mid 1)$. 


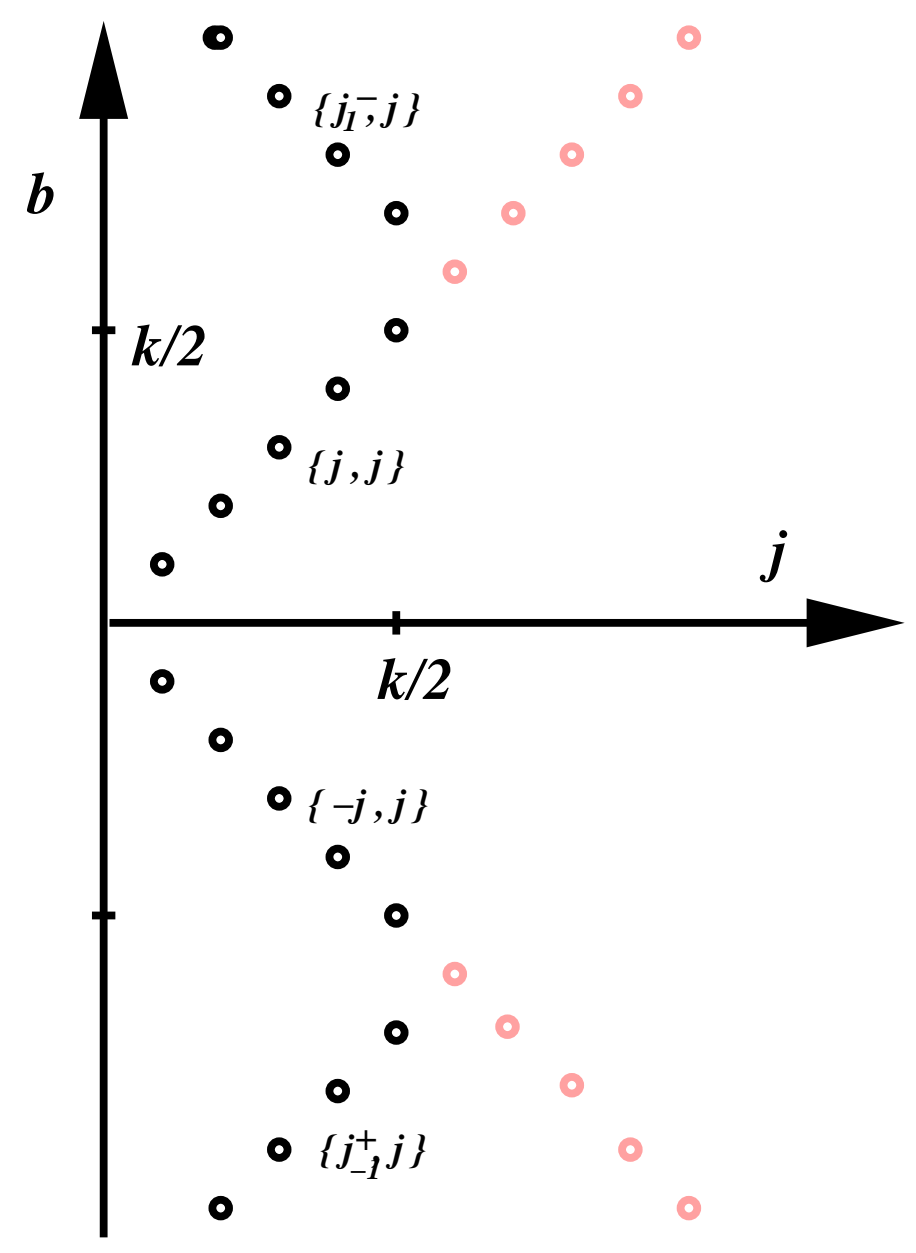

Figure 2: The set $A$ of atypical labels for the affine sl(2|1) algebra. Even though the $\operatorname{sl}(2)$ spin $j$ is cut off at $j=k / 2$, there exist infinitely many atypical labels (black dots) which are in one to one correspondence with the atypical labels of the finite dimensional algebra $\operatorname{sl}(2 \mid 1)$ (central black and pink dots). This correspondence is formalized by our map $\hat{\nu}$.

At first sight, the enumeration of atypical labels for our sl(2|1) current algebra may seem like a rather technical device. But there is more to it. We recall that atypical labels $\{ \pm j, j\}$ can also be enumerated by non-zero half-integers, i.e. $\nu(\{ \pm j, j\})= \pm j$. Our claim now is that the atypical class I representation with label $\left\{j_{m}^{ \pm}, j\right\}$ behaves very similarly to its finite dimensional counterpart $\nu^{-1} \circ \hat{\nu}\left(\left\{j_{m}^{ \pm}, j\right\}\right)$ in the sense that

$$
\chi_{\left\{j_{m}^{ \pm}, j\right\}}^{I}(q, z, \xi)=\chi_{\left\{\hat{\nu}\left(\left\{j_{m}^{ \pm}, j\right\}\right)\right\}}^{I I}(q, z, \xi)-\chi_{\left\{\hat{\nu}\left(\left\{ \pm j_{m}, j\right\}\right)-1 / 2 \operatorname{sgn} j_{m}^{ \pm}\right\}}^{I I}(q, z, \xi) .
$$

This formula is a rather central result for the representation theory of our current algebra. Let us stress that it is the affine version of a corresponding equality (2.7) between 
characters of $\operatorname{sl}(2 \mid 1)$ representations. As in the finite dimensional setup, eq. (3.4) emerges from the existence of fermionic singular vectors in atypical class I representations. In the case $m=0$, it claims that the only such singular vectors are those that appear in the atypical Kac-module spanned by the ground states. When $m \neq 0$, however, the ground states form a typical representation and the singular vectors appear only on the $|m|^{\text {th }}$ level of the class I module.

At first it may seem a bit surprising that affine representations $\left\{j_{m}^{ \pm}, j\right\}$ and $m \neq$ 0 behave so similarly to the Kac modules of $\operatorname{sl}(2 \mid 1)$. In the next subsection we shall understand this behavior in terms of spectral flow symmetries in the representation theory of the current algebra. Before any study of spectral flow automorphisms, it might be useful to illustrate the similarity between atypical representations of the current algebra and their finite dimensional counterpart more explicitly, at least for one example. To this end, let us focus on the representation $\{k / 2+1, k / 2\}^{\wedge}$ which we claim to be a close cousin of the sl $(2 \mid 1)$ representation $\{k / 2+1 / 2, k / 2+1 / 2\}$. By construction, the ground states of the current algebra representation transform in the typical multiplet $\{k / 2+1, k / 2\}$ and they possess conformal weight $h=-1$. From these vectors we can generate states with vanishing conformal weight with the help of modes in the current algebra. Such modes transform in the 8 -dimensional adjoint representation $\{0,1\}$ of $\operatorname{sl}(2 \mid 1)$. Through decomposition of the tensor product between $\{k / 2+1, k / 2\}$ and $\{0,1\}$ one finds that the Verma module over $\{k / 2+1 / 2, k / 2+1 / 2\}$ contains an atypical sl $(2 \mid 1)$ multiplet with conformal weight $h=0$. In fact, the results of [11] imply that the latter transforms according to the projective cover $\mathcal{P}(k / 2+1 / 2)$, see eq. (2.10). Not all of these states survive when we descend from the Verma module to the class I representation. This step involves removing bosonic singular vectors and a moment of reflections shows that such vectors with $h=0$ exist and that they transform in the submodule $\{k / 2+1, k / 2+1\}$ of $\mathcal{P}(k / 2+1 / 2)$. Hence, the states with $h=0$ in our class I representation decompose into the Kac-module $\{k / 2+1 / 2, k / 2+1 / 2\}$ plus a bunch of typical representations. The fermionic singular vectors that are responsible for eq. (3.4) transform in the subrepresentation $\{k / 2, k / 2\}$ of $\{k / 2+1 / 2, k / 2+1 / 2\}$, giving rise to the identity (3.4) with $j_{1}^{-}=k / 2+1$ and $j=k / 2$.

Before we draw some conclusions from eq. (3.4), let us quickly comment on our notations. Note that for $j=1 / 2$ and $m=0$ the above formula involves the character $\chi_{\{0\}}^{I I}$ of a representation which has a somewhat special status. In fact, it cannot be obtained as quotient of one of the indecomposable representations $\left\{j_{m}^{ \pm}, j\right\}^{\wedge}$, unlike the representations 
with characters $\chi_{\{n / 2\}}^{I I}, n \neq 0$. Instead, it arises as a submodule of the representations $\{ \pm 1 / 2,1 / 2\}^{\wedge}$. Our discussion suggest that $\chi_{\{0\}}^{I I}$ must be the character of the vacuum representation. In the terminology of Bowcock et al. the latter is a class IV representation. Thus, we shall also write $\chi_{\{0\}}^{I V}$ for this quantity.

Even though equation (3.4) is not a closed formula for the characters of class II representations, we can now use the same trick as in section 2.1.2 and write characters of class II representations as an infinite sum of class I characters,

$$
\chi_{\{ \pm j \mp 1 / 2\}}^{I I}(q, \xi, z)=-\sum_{n=0}^{\infty} \chi_{\hat{\nu}^{-1}( \pm j \pm n / 2)}^{I}(q, \xi, z)
$$

for $j=1 / 2,1,3 / 2, \ldots$ Note that the map $\hat{\nu}$ is invertible on all non-zero half-integers and it furnishes the label of the Kac module that sits at the bottom of the corresponding class I representation. By inserting our explicit formulas for class I characters we find

$$
\begin{aligned}
\chi_{\{j\}_{ \pm}}^{I I}(q, z, \xi)=-i \frac{\theta_{1}\left(z^{1 / 2} \xi^{-1 / 2}, q\right) \theta_{1}\left(z^{1 / 2} \xi^{1 / 2}, q\right)}{\eta^{3}(q) \theta_{1}(z, q)} \times \\
\quad \times \sum_{a \in \mathbb{Z}} q^{(k+1) a^{2} \mp 2 a j} \xi^{ \pm j}\left(\frac{z^{-a(k+1) \pm j}}{1+q^{a} z^{-1 / 2} \xi^{-1 / 2}}-\frac{z^{a(k+1) \mp j}}{1+q^{a} z^{1 / 2} \xi^{-1 / 2}}\right) .
\end{aligned}
$$

Character formulas of this type have to be used with some care: Before the denominators are expanded, one should spit the summation over $a$ into two parts. The one arising from positive values of $a$ can be converted into a power series right away. In all terms with non-negative $a$, however, one must first reduce the fraction by $q^{a}$ such that the subsequent expansion contains only non-negative powers of $q$. In the end, we recover the known results on the representation theory of the affine $\operatorname{sl}(2 \mid 1)$ algebra [5, 17]. Our

derivation was based on three ingredients: the decoupling formulas (3.1) for bosonic and fermionic generators, the structure (2.5) of atypical Kac modules for $\mathrm{sl}(2 \mid 1)$ and the fact that atypical class I representations with $m \neq 0$ decompose in the same way as in the case of $m=0$. We shall argue now that the last ingredient emerges from spectral flow symmetries in the representation theory of affine $\operatorname{sl}(2 \mid 1)$.

\subsection{Spectral flow symmetries}

The affine $\operatorname{sl}(2 \mid 1)$ algebra admits several interesting automorphisms. We shall be mainly concerned with two such spectral flow automorphisms $\gamma_{ \pm}$. By construction, $\gamma_{ \pm}$are defined 
on the entire current algebra, but for our purposes it is sufficient to know how they act on the generators $B_{0}, H_{0}, L_{0}$,

$$
\gamma_{ \pm}\left(B_{0}\right)=B_{0} \pm k / 2 \quad, \quad \gamma_{ \pm}\left(H_{0}\right)=H_{0}+k / 2 \quad, \quad \gamma_{ \pm}\left(L_{0}\right)=L_{0}+H_{0} \mp B_{0}
$$

From these formulas we may infer how (super-)characters behave under the action of $\gamma_{ \pm}$and this in turn is sufficient to determine how spectral flow automorphisms map representations of the current algebra onto each other. Along with $\gamma_{ \pm}$we shall also be interested in the composite automorphism $\gamma=\gamma_{+} \gamma_{-}^{-1}$ which acts as

$$
\gamma\left(B_{0}\right)=B_{0}+k \quad, \quad \gamma\left(H_{0}\right)=H_{0} \quad, \quad \gamma\left(L_{0}\right)=L_{0}-2 B_{0}-k
$$

Any automorphisms of the current algebra gives rise to a map between representations and hence to a map between characters. From the action on the zero modes $B_{0}, H_{0}$ and $L_{0}$ we can easily read off that

$$
\gamma_{ \pm} \chi(q, \xi, z)=\xi^{ \pm k / 2} z^{k / 2} \chi\left(q, q^{\mp 1} \xi, q z\right) \quad, \quad \gamma \chi(q, \xi, z)=\xi^{k} q^{-k} \chi\left(q, q^{-2} \xi, z\right)
$$

for all characters $\chi$ of the $\operatorname{sl}(2 \mid 1)$ current algebra. If $\mathcal{R}$ is any representation of $\widehat{\operatorname{sl}}(2 \mid 1)$ and $\chi_{\mathcal{R}}$ is its character, then the image $\sigma \mathcal{R}$ of $\mathcal{R}$ under an automorphism $\sigma$ obeys

$$
\sigma \chi_{\mathcal{R}}(q, \xi, z)=\chi_{\sigma \mathcal{R}}(q, \xi, z)
$$

Given the character $\chi_{\mathcal{R}}$ of some representation $\mathcal{R}$, we can use eqs. (3.7) to compute its image under the above automorphisms $\sigma=\gamma_{ \pm}, \gamma$. This in turn allows us to recover uniquely the representations $\gamma_{ \pm} \mathcal{R}$ and $\gamma \mathcal{R}$.

In the following we shall spell out the action of our spectral flow automorphisms on the class I and II representations we have studied above. Our rather compact notations allow us to summarize the results for the spectral flow automorphisms $\gamma_{ \pm}$in a single line

$$
\gamma_{ \pm}\left(\{b, j\}^{\wedge}\right)=\left\{b \pm k / 2 \pm \frac{1}{2}, k / 2+\frac{1}{2}-j\right\}^{\wedge}, \gamma_{ \pm}\left(\{n / 2\}^{\wedge}\right)=\{n / 2 \pm k / 2\}^{\wedge}
$$

To verify our assertions, the reader is invited to convert them into identities between super-characters and to check these identities by direct computation. The formulas become somewhat more explicit if we label irreducible representations according to the 
representation their ground states transform in,

$$
\begin{array}{ll}
\{b, j\} \stackrel{\gamma_{ \pm}}{\longrightarrow}\left\{b \pm k / 2 \pm \frac{1}{2}, k / 2+\frac{1}{2}-j\right\} & \text { for } \quad b \neq \pm(j-k-1) \\
\{b, j\} \stackrel{\gamma_{ \pm}}{\longrightarrow}\left\{k / 2+\frac{1}{2}-j\right\}_{\mp} & \text { for } b= \pm(j-k-1) \\
\{j\}_{ \pm} \stackrel{\gamma_{ \pm}}{\longrightarrow}\left\{ \pm\left(j+k / 2+\frac{1}{2}\right), k / 2+\frac{1}{2}-j\right\} & \text { for } j \neq 0 \\
\{j\}_{\mp} \stackrel{\gamma_{ \pm}}{\longrightarrow}\{k / 2-j\}_{ \pm}, \quad\{0\} \stackrel{\gamma_{ \pm}}{\longrightarrow}\{k / 2\}_{ \pm} .
\end{array}
$$

The third line, for example, tells us that the image of the irreducible representation generated from the atypical representation $\{j\}_{+}$under the action of $\gamma_{+}$is an irreducible representation whose ground states transform in the typical representation $\{j+k / 2+$ $\left.\frac{1}{2}, k / 2+\frac{1}{2}-j\right\}$. The latter may be obtained from the corresponding Verma module by removing singular vectors on some excited levels.

We also want to spell out analogous formulas for the automorphism $\gamma=\gamma_{+} \circ \gamma_{-}^{-1}$. In the compact notation, its action is given by

$$
\gamma\left(\{b, j\}^{\wedge}\right)=\{b+k+1, j\}^{\wedge}, \gamma\left(\{n / 2\}^{\wedge}\right)=\{n / 2+k\}^{\wedge}
$$

Note that the symmetry $\gamma$ maps sectors whose ground states transform in an atypical representation $\{j\}_{ \pm}$of the Lie superalgebra $\operatorname{sl}(2 \mid 1)$ into sectors with typical spaces of ground states according to the following rules,

$$
\begin{aligned}
& \{j\}_{ \pm} \stackrel{\gamma^{m}}{\longrightarrow}\left\{j_{m}^{ \pm}, j\right\} \text { for } \pm m>0 \\
& \{j\}_{ \pm} \stackrel{\gamma^{m}}{\longrightarrow}\left\{j_{m}^{ \pm} \pm 1 / 2, j+1 / 2\right\} \text { for } \pm m<0 .
\end{aligned}
$$

Hence, the existence of the spectral flow symmetries explains why the representations $\left\{j_{m}^{ \pm}, j\right\}$ behave like atypical representations of the affine $\mathrm{sl}(2 \mid 1)$ algebra: they are simply related to the sectors erected over atypical sl(2|1) representations by an automorphism.

\subsection{Modular transformation and S-matrix}

We would like to conclude this section on the representation theory of the $\operatorname{sl}(2 \mid 1)$ current algebra with a few comments on modular properties of the characters. In the following we

shall consider the characters as functions of $\tau, \sigma$ and $\nu$. They are related to the variables we used above through $q=\exp 2 \pi i \tau, z=\exp 2 \pi i \sigma$ and $\xi=\exp 2 \pi i \nu$, as usual. From 
our explicit formula (3.2) for characters of class I representations it is easy to infer the auxiliary formula

$$
\chi_{\{b=0, j\}}^{I}\left(-\frac{1}{\tau}, \frac{\sigma}{\tau}, \frac{\nu}{\tau}\right)=-\frac{1}{\sqrt{-i \tau}} e^{\frac{i \pi k}{2 \tau} \sigma^{2}} e^{\frac{i \pi}{2 \tau} \nu^{2}} \sum_{j^{\prime}=1 / 2}^{\frac{k}{2}} \sqrt{\frac{2}{k+1}} \sin \frac{4 \pi j j^{\prime}}{k+1} \chi_{\left\{b=0, j^{\prime}\right\}}^{I}(\tau, \sigma, \nu) .
$$

Note that the right hand side contains an explicit $\tau$ dependence which, if we demand that the modular transform be interpreted in a conventional sense and closes onto characters, suggests the contribution of a continuous spectrum of exponents. The need is confirmed by the modular transformation of the character for $\{b, j\}$ representations with $b \neq 0$, which require an integral representation of $e^{2 i \pi \tau b^{2} /(k+1)}$ etc. After some Gaussian integration, we find

$$
\chi_{\{b, j\}}^{I}\left(-\frac{1}{\tau}, \frac{\sigma}{\tau}, \frac{\nu}{\tau}\right)=i e^{\frac{i \pi k}{2 \tau}\left(\sigma^{2}-\nu^{2}\right)} \sum_{j^{\prime}=1 / 2}^{\frac{k}{2}} \frac{2}{k+1} \sin \frac{4 \pi j j^{\prime}}{k+1} \int_{-\infty}^{\infty} d b^{\prime} e^{\frac{4 i \pi b b^{\prime}}{k+1}} \chi_{\left\{b^{\prime}, j^{\prime}\right\}}^{I}(\tau, \sigma, \nu)
$$

where we formally evaluated integrals of the type $\int \exp \left(-i \tau x^{2}\right)=\sqrt{\pi / i \tau}$ [of course, the integrals are naively divergent as $\operatorname{Im} \tau>0$.]

Modular transformations of the type II and IV characters are a bit more cumbersome to work out. It can be attacked rather efficiently using our representations (3.5) as infinite sums of class I characters. Here we shall content ourselves with the example of the class IV representation at $k=1$. If we also set $\sigma=\nu=0$ we find that

$$
\chi_{\{0\}}^{I V}(-1 / \tau)=-\frac{i}{2} \int_{-\infty}^{\infty} \frac{d b^{\prime}}{\cos \pi b^{\prime}} \chi_{\left\{b^{\prime}, 1 / 2\right\}}^{I}(\tau)
$$

where the contour has to avoid the poles. Rotating into the purely imaginary direction gives a contribution from poles which is easily identified with $\chi_{\{0\}}^{I V}$. The remaining integral can be factored in terms of $\chi_{\{0,1 / 2\}}^{I}$,

$$
\chi_{\{0\}}^{I V}(-1 / \tau)=-\chi_{\{0\}}^{I V}(\tau)+\int d \alpha \frac{q^{\frac{\alpha^{2}}{2}}}{\cosh \pi \alpha} \chi_{\{0,1 / 2\}}^{I}(\tau)
$$

We thus recover by this very elementary means the results of [5] obtained through use of the Mordell integral [19]. The construction of modular invariants using these transformation formulas is a complex problem, which we shall address later in the case $k=1$. 


\section{The state space and partition functions}

Our aim now is to formulate a proposal for the states space of the sl(2|1) WZNW model. We shall then verify our claim in the special case $k=1$ through a free field representation of the model. The third subsection is devoted to the partition function of the theory. The latter forgets all information about the complicated way in which irreducible blocks are glued together to build $\mathcal{J}$. We then specialize once more to $k=1$ and comment on the global topology of the target space.

\subsection{The proposal for integer level $k$}

It is now rather straightforward to come up with a proposal for the state space of the WZNW model on $\mathrm{SU}(2 \mid 1)$. In fact, we can simply depart from formula (2.18) and make it symmetric with respect to the action of our spectral flow symmetry. The invariance under the action of $\gamma$ should be considered as an additional input. In principle, the spectral flow symmetry of the $\operatorname{sl}(2 \mid 1)$ current algebra could be broken by the physical couplings of the theory. Since this did not happen for the GL(1|1) WZNW model, it seems natural to propose

$$
\mathcal{H}_{C F T}=\sum_{j=1 / 2}^{k / 2} \sum_{b \neq j_{m}^{ \pm}}\{b, j\}_{L}^{\wedge} \otimes\{-b, j\}_{R}^{\wedge} \oplus \mathcal{J}^{\wedge}
$$

where $\mathcal{J}^{\wedge}$ is a single indecomposable representation of the two (anti-)commuting super current algebras that contains all the atypical contributions. It is composed from the atypical building blocks $\left\{l_{1}\right\} \otimes\left\{l_{2}\right\}$ in the same way as in the minisuperspace theory. To obtain the corresponding diagram one simply has to replace $\{j\}_{ \pm}=\{ \pm j\}$ with $\{ \pm j\}^{\wedge}$.

By construction, all the $\operatorname{sl}(2 \mid 1)$ currents act on the state space (4.1) and they obey periodic boundary conditions. This applies in particular to the fermionic fields. One can find a second, closely related theory in which only bosonic fields are periodic. In order to construct its state space, we need to revisit our discussion of spectral flow symmetries. As we have mentioned above, the automorphisms we have investigated in the previous section all extend to the entire current algebra. In particular, they map fermionic modes with integer mode numbers onto each other, i.e. they respect periodic boundary conditions on the fermionic currents. There exists yet another important isomorphism that intertwines

between integer and half-integer mode numbers for the fermionic generators. It can be considered as the square root of the automorphism $\gamma$. On the bosonic zero modes, the 
new isomorphism $\vartheta$ is given by

$$
\vartheta\left(B_{0}\right)=B_{0}+k / 2 \quad, \quad \vartheta\left(H_{0}\right)=H_{0} \quad, \quad \vartheta\left(L_{0}\right)=L_{0}-B_{0}-k / 2 .
$$

$\vartheta$ extends to the full current algebra such that it acts trivially on the bosonic sl(2) currents and it shifts modes of the fermionic currents by $\pm 1 / 2$, as usual.

Our isomorphism $\vartheta$ induces a map between representations of the current algebra with integer fermionic modes and a new type of representations in which fermionic generators have half integer mode numbers. According to the usual terminology, the former class of representations form the $\mathrm{R}$ sector while the latter belong to the NS sector of the theory. The theory with state space (4.1) includes exclusively $\mathrm{R}$ sector representations in which all currents obey periodic boundary conditions. Another option is to consider a theory that encompasses both R and NS sector with the state space given by

$$
\tilde{\mathcal{H}}_{C F T}=\mathcal{H}_{C F T}^{R} \oplus \mathcal{H}_{C F T}^{N S}=\mathcal{H}_{C F T} \oplus \vartheta \mathcal{H}_{C F T}
$$

Note that the NS sector has exactly the same intricate structure as the R sector since the former is the image of the latter under the action of $\theta$. In the following we shall refer to both models as WZNW model on $\mathrm{SU}(2 \mid 1)$. Even though it seems natural to include the NS sector, it is not required by all applications.

\subsection{Free field representation at $k=1$}

So far, the main motivation for our proposal (4.1) came from the harmonic analysis on $\mathrm{SU}(2 \mid 1)$. By construction, we are guaranteed to recover the correct state space of the particle limit when we send the level $k$ to infinity. Our formula (4.1) applies to finite $k$ and it suggest that field theory effects would merely truncate the spin $j$ to an value $j \leq k / 2$ and then make the whole theory symmetric with respect to spectral flow. We are now going to test this proposal in the extreme quantum case, namely at $k=1$. At this point, the WZNW model admits a free field representation that we are going to spell out momentarily. It involves a pair of symplectic fermions $\eta_{1}, \eta_{2}$, and a pair of bosons $\phi, \phi^{\prime}$. While the boson $\phi$ comes with the usual metric, $\phi^{\prime}$ is assumed to be time-like. For their propagators this means

$$
\begin{aligned}
\left\langle\eta_{1}(z, \bar{z}) \eta_{2}(w, \bar{w})\right\rangle & =-\ln |z-w|^{2} \\
\langle\phi(z, \bar{z}) \phi(w, \bar{w})\rangle & =-\ln |z-w|^{2} \\
\left\langle\phi^{\prime}(z, \bar{z}) \phi^{\prime}(w, \bar{w})\right\rangle & =\ln |z-w|^{2} .
\end{aligned}
$$


Note that the central charge of this free field theory is $c=-2+1+1=0$ and hence it agrees with the central charge of $\mathrm{SU}(2 \mid 1)$ WZNW models. We shall begin our discussion of the WZNW model with explicit formulas for the currents. In order to construct the four bosonic currents, we need to split the space-like bosonic field $\phi(z, \bar{z})=\varphi(z)+\bar{\varphi}(\bar{z})$ into its chiral components. Our bosonic currents then read,

$$
\begin{aligned}
E^{+}(z)=e^{\sqrt{2} i \varphi(z)} & , & E^{-}(z) & =e^{-\sqrt{2} i \varphi(z)} \\
H(z)=\frac{1}{\sqrt{2}} i \partial \phi(z) & & B(z) & =-\frac{1}{\sqrt{2}} i \partial \phi^{\prime}(z) .
\end{aligned}
$$

The necessity to split $\phi$ into its chiral components means that the boson $\phi$ is compactified to the so-called self-dual radius, as usual in the free field representation of the $\mathrm{SU}(2)$ WZNW model at level $k=1$. In addition, the following expressions for the four fermionic currents also involve the chiral components $\varphi^{\prime}$ and $\bar{\varphi}^{\prime}$ of the time-like bosonic field $\phi^{\prime}(z, \bar{z})=\varphi^{\prime}(z)+\bar{\varphi}^{\prime}(\bar{z})$,

$$
V^{ \pm}(z)=e^{\frac{1}{\sqrt{2}} i\left( \pm \varphi(z)+\varphi^{\prime}(z)\right)} \partial \eta_{1}(z) \quad, \quad W^{ \pm}(z)=e^{\frac{1}{\sqrt{2}} i\left( \pm \varphi(z)-\varphi^{\prime}(z)\right)} \partial \eta_{2}(z)
$$

Similarly, one may spell out the anti-holomorphic generators of the sl(2|1) current algebra. It is rather easy to check that the above expressions give rise to fields with the correct operator product expansions. Let us note that the free field representation we consider in this section has to be distinguished clearly from the Kac-Wakimoto type construction (3.1) we have used earlier to construct the characters at integer levels $k$. We shall comment on this a bit more later on.

It is possible to check that fields of dimension zero can be organized exactly as it is suggested by our diagram (2.19). We shall just sketch the relevant arguments because a full proof is rather laborious to write down. Let us consider the left part of the diagram only and identify the fields that make up the various blocks of the composition series. Clearly, the $\{0\} \times\{0\}$ representation at the top corresponds to the field $\eta_{1} \eta_{2}$. From here we can act with the fermionic currents $W^{ \pm}, \bar{V}^{ \pm}$and arrive at expressions for the two blocks on the intermediate level of the diagram,

$$
\begin{aligned}
& \{1 / 2\}_{-} \times\{0\}: \quad e^{ \pm i \varphi / \sqrt{2}} e^{-i \varphi^{\prime} / \sqrt{2}} \eta_{2}, \quad e^{-i \sqrt{2} \varphi^{\prime}} \eta_{2} \partial \eta_{2}, \\
& \{0\} \times\{1 / 2\}_{+}: \quad e^{ \pm i \bar{\varphi} / \sqrt{2}} e^{i \bar{\varphi}^{\prime} / \sqrt{2}} \eta_{1} \quad, \quad e^{i \sqrt{2} \bar{\varphi}^{\prime}} \eta_{1} \bar{\partial} \eta_{1} .
\end{aligned}
$$


From the previous formulas we can read off the fields that make up the topmost representation $\{1 / 2\}_{-} \times\{1 / 2\}_{+}$in our diagram,

$$
\{1 / 2\}_{-} \times\{1 / 2\}_{+}: \quad\left\{\begin{array}{c}
e^{ \pm i \varphi / \sqrt{2}} e^{-i \varphi^{\prime} / \sqrt{2}} \eta_{2} \\
e^{-i \sqrt{2} \varphi^{\prime}} \eta_{2} \partial \eta_{2}
\end{array} \times\left\{\begin{array}{c}
e^{ \pm i \bar{\varphi} / \sqrt{2}} e^{i \bar{\varphi}^{\prime} / \sqrt{2}} \eta_{1} \\
e^{i \sqrt{2} \bar{\varphi}^{\prime}} \eta_{1} \bar{\partial} \eta_{1} .
\end{array}\right.\right.
$$

Acting with the holomorphic fermionic currents $V^{ \pm}(z)$ we arrive at the following formulas for fields that belong to the multiplet

$$
\{1\}_{-} \times\{1 / 2\}_{+}: \quad\left\{\begin{array}{c}
\left( \pm \partial \phi+\partial \phi^{\prime}\right) \eta_{2} \partial \eta_{2} e^{ \pm i \varphi / \sqrt{2}} e^{-3 i \varphi^{\prime} / \sqrt{2}} \\
\eta_{2} e^{ \pm i \sqrt{2} \varphi} e^{-i \sqrt{2} \varphi^{\prime}} \\
\eta_{2} \partial \phi e^{-i \sqrt{2} \varphi^{\prime}}
\end{array} \times\left\{\begin{array}{c}
e^{ \pm i \bar{\varphi} / \sqrt{2}} e^{i \bar{\varphi}^{\prime} / \sqrt{2}} \\
e^{i \sqrt{2} \bar{\varphi}^{\prime}} \bar{\partial} \eta_{1}
\end{array} .\right.\right.
$$

on the intermediate level of the diagram. Our notation means that every product of the three holomorphic fields on the left hand side with the three anti-holomorphic fields on the right hand side is part of this 9-dimensional block. Similarly, we can now descend to the bottom of the diagram,

$$
\{1 / 2\}_{-} \times\{1 / 2\}_{+}: \quad\left\{\begin{array}{c}
e^{ \pm i \varphi / \sqrt{2}} e^{-i \varphi^{\prime} / \sqrt{2}} \\
\eta_{2} \partial \phi^{\prime} e^{-i \sqrt{2} \varphi^{\prime}}
\end{array} \times\left\{\begin{array}{c}
e^{ \pm i \bar{\varphi} / \sqrt{2}} e^{i \bar{\varphi}^{\prime} / \sqrt{2}} \\
e^{i \sqrt{2} \bar{\varphi}^{\prime}} \bar{\partial} \eta_{1}
\end{array}\right.\right.
$$

Finally, the representation $\{0\} \times\{0\}$ in center bottom position is represented by the identity field. It is easy but laborious to check that the different representations are connected by the action of the left and right generators as indicated in the diagram. In checking this, notice that $\eta_{2} \partial \phi^{\prime} e^{-i \sqrt{2} \varphi^{\prime}} \equiv \partial \eta_{2} e^{-i \sqrt{2} \varphi^{\prime}}$ up to a total derivative.

There are a number of interesting further comments and observations that we would like to make. Let us begin with a brief comment on the relation with Kac-Wakimoto like representations of the form (3.1). As discussed in [8], a naive evaluation of the action of the latter on vertex operators leads to a much simpler picture in which the atypical sector is a smooth deformation of the typical part. In particular, there is no mixing between left- and right movers as in the case of the representation $\mathcal{J}^{\wedge}$. In order to see the latter, the screening charge of the Kac-Wakimoto representation must be taken into account (see [8] for details). The free field representation we have employed in this subsection is much simpler to use, but it is restricted to $k=1$.

The free field representation also allows us to illustrate very explicitly how atypical fields of dimension $h=0$ are embedded into sectors with ground states in typical multiplets once their spin exceeds $k / 2$. Take, for instance, the field $O=e^{i \sqrt{2}\left(\varphi-\varphi^{\prime}\right)} \eta_{2}$ from the 
$\{1\}_{-}$representation and observe that

$$
E^{-}(z) e^{i \sqrt{2}\left(\varphi-\varphi^{\prime}\right)} \eta_{2}(w)=\frac{1}{(z-w)^{2}}: e^{-i \sqrt{2} \phi(z)} e^{i \sqrt{2}\left(\varphi-\varphi^{\prime}\right)} \eta_{2}(w):+\ldots .
$$

Thus $O$ is not a highest weight of the current algebra, and applying a current operator can give rise to a field of dimension $h=-1$, namely the field $P=e^{-i \sqrt{2} \varphi^{\prime}} \eta_{2}$. This field belongs

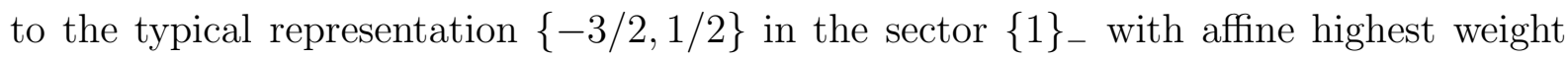
$Q=e^{i\left(\varphi-3 \varphi^{\prime}\right) / \sqrt{2}} \eta_{2} \partial \eta_{2}$. One may easily generalize these observations to all representations in the complex, hence confirming our analysis based on spectral flow.

Let us finally come to the most important point, which concerns the possible construction of consistent theories 3 that are realized on a subspace of our state space (4.1). Note that $\mathcal{H}$ does contain a large number of fermionic singular vectors that we decided not do decouple, partly because the minisuperspace analysis suggested that it was unnatural to do so. But to a certain extend one should consider (4.1) as some kind of maximal choice from which other models can be obtained by consistent decoupling of singular vectors. In general there can be several such reduced theories. Once more we may use our free field representation for the $k=1$ model to illustrate nicely how this works. Note that the expressions (4.4) for the currents only contain derivatives of the fermionic fields. Hence, we do not spoil the sl(2|1) current algebra symmetry if we decide to work with a model in which the fundamental fields are e.g. $\phi, \phi^{\prime}, \eta_{2}$ and $\partial \eta_{1}$. Since $\eta_{1}$ is not part of this model, some of the sectors we discussed above do no longer appear. This concerns the sectors $\{0\} \times\{0\}$ and $\{1 / 2\}_{-} \times\{1 / 2\}_{+}$on the top floor of $\mathcal{J}^{\wedge}$ and the sector $\{0\} \times\{1 / 2\}_{+}$on the intermediate floor. In the resulting model, there is still a single atypical sector that comprises all the irreducible atypicals, but it is reduced to two floors and has the shape of a saw blade. Obviously, a similar analysis applies to the theory that contains $\partial \eta_{2}$ instead of $\eta_{2}$. But we can even go one step further and drop both $\eta_{1}$ and $\eta_{2}$ so that only fermionic derivatives remain. What results is a model whose atypical sector decomposes into an infinite sum of irreducibles. The latter are the sectors that appear on the bottom floor of $\mathcal{J}^{\wedge}$, i.e. $\{0\} \times\{0\}$ and $\{1 / 2\}_{-} \times\{1 / 2\}_{+}$from our list above. All others need either $\eta_{1}$ or $\eta_{2}$. Similar phenomena are possible at other levels. We shall see another explicit example in section 5.2. Let us stress, however, that the free field construction at $k=1$ does not include the "defining" $\{0,1 / 2\}$ field of the WZNW model. A careful study of

\footnotetext{
${ }^{3}$ Consistency in this paragraph refers to the existence of genus zero correlators obeying the usual factorization constraints. The construction and behavior of torus amplitudes is not addressed.
} 
the Knizhnik-Zamolodchikov equations [3, 2] shows that consistency in the presence of the $\{0,1 / 2\}$ sector requires the identity field to be embedded into an indecomposable sector. In this sense, the fully truncated atypical sector we have just described cannot be embedded into the sl(2|1) WZNW model.

\subsection{Partition functions}

We now go back to the full theory based on our proposal (4.1). We would like to compute the partition function of the model, with and without inclusion of the NS sector. Since

partition functions are obtained by taking the trace over the state space, the details of the action of fermionic generators in the atypical sector $\mathcal{J}^{\wedge}$ do not show up in the result. In other words, the contribution from the indecomposable $\mathcal{J}^{\wedge}$ is the same as if we would take the trace over a sum of its irreducible components. The latter can be resumed as follows,

$$
\begin{aligned}
\operatorname{str}_{\mathcal{J}^{\wedge}} q^{L_{0}+\bar{L}_{0}}= & \sum_{\nu \in \mathbb{Z} / 2} 2 \chi_{\{\nu\}}^{I I}(\bar{q}) \chi_{\{-\nu\}}^{I I}(q)-\chi_{\{\nu+1 / 2\}}^{I I}(\bar{q}) \chi_{\{-\nu\}}^{I I}(q)-\chi_{\{\nu-1 / 2\}}^{I I}(\bar{q}) \chi_{\{-\nu\}}^{I I}(q) \\
= & \sum_{\nu \in \mathbb{Z} / 2}\left(\chi_{\{\nu\}}^{I I}(\bar{q})-\chi_{\{\nu-1 / 2\}}^{I I}(\bar{q})\right)\left(\chi_{\{-\nu\}}^{I I}(q)-\chi_{\{-\nu+1 / 2\}}^{I I}(q)\right) \\
= & \sum_{\nu=1 / 2,1, \ldots}\left(\chi_{\{\nu\}}^{I I}(\bar{q})-\chi_{\{\nu-1 / 2\}}^{I I}(\bar{q})\right)\left(\chi_{\{-\nu\}}^{I I}(q)-\chi_{\{-\nu+1 / 2\}}^{I I}(q)\right) \\
& +\sum_{\nu=1 / 2,1, \ldots}\left(\chi_{\{-\nu\}}^{I I}(\bar{q})-\chi_{\{-\nu+1 / 2\}}^{I I}(\bar{q})\right)\left(\chi_{\{\nu\}}^{I I}(q)-\chi_{\{\nu-1 / 2\}}^{I I}(q)\right) \\
= & \sum_{ \pm} \sum_{m \in \mathbb{Z}} \sum_{j=1 / 2}^{k / 2} \chi_{\{j m, j\}}^{I}(\bar{q}) \chi_{\{-j m, j\}}^{I}(q) .
\end{aligned}
$$

In the last step we have inserted the relation (3.4) between characters of class I and class II representations and we used the isomorphism $\hat{\nu}$ to convert the sum over non-zero half-integers into a sum over $m$ and $j$. Our result shows that the contribution from the atypical representations agrees exactly with the part that we omitted from the typical sector of the theory. Hence, the full partition function becomes

$$
Z(q)=\sum_{j=1 / 2}^{k / 2} \sum_{b \in \mathbb{R}} \chi_{\{b, j\}}^{I}(\bar{q}) \chi_{\{-b, j\}}^{I}(q)
$$


Let us also briefly discuss how the partition functions is modified when we want to include the NS sector. In that case, the trace extends over both $\mathcal{H}_{C F T}$ and its image under the spectral flow $\vartheta$. The modular invariant partition function $\tilde{Z}(q)$ of this theory contains four different terms, two in which $(-1)^{F}$ is inserted and two in which it is not. It is customary to label the corresponding contributions with $R, s R, N S$ and $s N S$ where the small $s$ signals the insertion of $(-1)^{F}$. With these notations, the standard super-characters we have discussed throughout the previous section should all carry a superscript $s R$. It is easy to find explicit formulas for the other three sets of (super-)characters using the relation

$$
\chi^{s N S}(q, \xi, z)=\vartheta \chi^{s R}(q, \xi, z)=\xi^{k / 2} q^{-k / 2} \chi^{s R}\left(q, q^{-1} \xi, z\right)
$$

to convert $s R$ characters into $s N S$ super-characters. The same prescription is used when we construct $N S$ characters from the $\mathrm{R}$ sector, only that we have to replace the $R$ supercharacters $\chi$ by ordinary characters. The partition function $\tilde{Z}(q)$, finally, has the same form as eq. (4.9) with an additional summation over all four types of terms.

Let us illustrate the previous results in the case of $k=1$ again. The $(s R)$ characters of this theory take a particularly simple form, as was first observed by Bowcock et al. in [5],

$$
\chi_{\{b, 1 / 2\}}^{I}(q, z, \xi)=\frac{q^{-b^{2} / 2} \xi^{b}}{\eta(q)}\left[\chi_{1 / 2}(q, \xi) \chi_{0}(q, z)-\chi_{0}(q, \xi) \chi_{1 / 2}(q, z)\right]
$$

where $\chi_{0}$ and $\chi_{1 / 2}$ are $S U(2)$ level one characters for spin 0 and $1 / 2$ respectively. This expression allows us to determine the modular invariant physical partition function $\tilde{Z}$ involving periodic or antiperiodic boundary conditions for the fermions along both periods of the torus. The doubly periodic sector $\left(\chi^{s R}\right)$ gives a vanishing contribution for characters $\chi_{\{b, 1 / 2\}}$ since the super-dimension of the horizontal Kac modules vanishes. We are left with three contributions, which read respectively

$$
\begin{aligned}
\chi_{\{b, 1 / 2\}}^{R}(q) & =2 \frac{q^{-b^{2} / 2}}{\eta(q)} \chi_{1 / 2}(q) \chi_{0}(q) \\
\chi_{\{b, 1 / 2\}}^{N S}(q) & =\frac{q^{-b^{2} / 2}}{\eta(q)}\left[\chi_{0}^{2}(q)+\chi_{1 / 2}^{2}(q)\right] \\
\chi_{\{b, 1 / 2\}}^{s N S}(q) & =\frac{q^{-b^{2} / 2}}{\eta(q)}\left[\chi_{0}^{2}(q)-\chi_{1 / 2}^{2}(q)\right] .
\end{aligned}
$$


Their modular transformations are easy to obtain for $b=0$

$$
\begin{aligned}
& \chi_{\{0,1 / 2\}}^{R}(-1 / \tau)=\frac{1}{\sqrt{-i \tau}} \chi_{\{0,1 / 2\}}^{s N S}(\tau) \\
& \chi_{\{0,1 / 2\}}^{N S}(-1 / \tau)=\frac{1}{\sqrt{-i \tau}} \chi_{\{0,1 / 2\}}^{N S}(\tau) \\
& \chi_{\{0,1 / 2\}}^{s N S}(-1 / \tau)=\frac{1}{\sqrt{-i \tau}} \chi_{\{0,1 / 2\}}^{R}(\tau) .
\end{aligned}
$$

Obviously, the $\tau$ dependence of these formulas originates in the $1 / \eta$ term in the characters, and has to be compensated by a similar factor coming from the $b$ sum in order to obtain a modular invariant quantity.

The question we want to ask now is what kind of sum over $b$ one should consider. It seems at first sight that, since we are dealing with $\mathrm{SU}(2 \mid 1)$, the $b$ number should be discrete, in agreement with the imaginary exponential appearing in section 2.2 (note that this is also compatible with invariance under action of the spectral flow). It is likely that such a theory would make sense in genus zero. Difficulties arise, however, when we try to establish modular invariance of the partition function, i.e. consider the theory in genus one. Indeed, even if $b$ is discretized, there is no reason to truncate its range, and thus the naive spectrum of conformal weights is unbounded from below, and exhibits arbitrarily large negative dimensions. This should not come as a surprise since the metric on the group is not positive definite, and thus the naive functional integral in the WZNW model is divergent. What is required to obtain a physical partition function on the torus - one that could be compared with the spectrum of some lattice Hamiltonian say - is some sort of analytic continuation.

This raises some interesting questions on which we would like to digress briefly. For a compactified time-like boson, partition functions would involve sums of the form

$$
\sum_{n} e^{\lambda n^{2}}, \quad \operatorname{Re} \lambda>0
$$

This sum is obviously divergent, but one could be tempted to give it a meaning by analytical continuation from a similar sum with $\operatorname{Re} \lambda<0$. An equivalent problem arises when we try to continue a theta function such as

$$
\theta(\tau)=\sum_{n} e^{i \pi \tau n^{2}}
$$


into the lower half plane $\operatorname{Im} \tau<0$. It is known that this continuation is not possible, since the $\theta$ function has singularities which are dense on the real axis (a quick proof is obtained by first observing that $\theta$ is singular for $\tau$ an even integer, and then using modular transformations). Theta functions have a natural boundary, and are simple examples of lacunary functions, i.e. "almost all" their Fourier coefficients are zero [20]. The partition function of a compactified time-like boson is thus a formal object from which it is hard to extract physical meaning. On the other hand, without compactification, the partition function can easily be analytically continued. Indeed, replacing the discrete sum by an integral we have

$$
\int_{-\infty}^{\infty} d x e^{i \pi \tau x^{2}}=\frac{1}{\sqrt{-i \tau}}
$$

which can be continued in the lower half plane since it has a single cut along the negative imaginary axis.

We thus restrict to the theory with continuous spectrum of $b$, and propose the simplest partition function

$$
Z_{k=1}=\left(\left|\chi_{\{0,1 / 2\}}^{R}\right|^{2}+\left|\chi_{\{0,1 / 2\}}^{N S}\right|^{2}+\left|\chi_{\{0,1 / 2\}}^{s N S}\right|^{2}\right) \int_{-\infty}^{\infty} d b(q \bar{q})^{-b^{2} / 2}
$$

which we interpret through analytic continuation, up to an irrelevant phase, as

$$
\begin{aligned}
Z_{k=1}^{\text {phys }} & =\left(\left|\chi_{\{0,1 / 2\}}^{R}\right|^{2}+\left|\chi_{\{0,1 / 2\}}^{N S}\right|^{2}+\left|\chi_{\{0,1 / 2\}}^{s N S}\right|^{2}\right) \int_{-\infty}^{\infty} d \alpha(q \bar{q})^{\alpha^{2} / 2} \\
& =\left(\left|\chi_{0}\right|^{2}+\left|\chi_{1 / 2}\right|^{2}\right)^{2} \times \frac{1}{\eta \bar{\eta}} \int_{-\infty}^{\infty} d \alpha(q \bar{q})^{\alpha^{2} / 2}
\end{aligned}
$$

This object is obviously modular invariant since, from a direct calculation of the integrals,

$$
\int d \alpha(q \bar{q})^{\alpha^{2} / 2}=\frac{1}{\sqrt{\tau \bar{\tau}}} \int d \alpha(\tilde{q} \tilde{\tilde{q}})^{\alpha^{2} / 2}
$$

hence compensating the factors coming from the $\eta$ functions in the characters. We note that the spectrum of conformal weights in the periodic sector is a continuum starting at $h=1 / 8$. The field with $h=0$ does not appear.

In conclusion, the requirement for our theory to possess a "physical partition function" has forced us to let $b$ be continuous. Geometrically, this amounts to a decompactification of the time-like circle. Hence we are led to consider the universal cover of $\mathrm{SU}(2 \mid 1)$ so that we can perform an analytical continuation on the number $b$. One may interpret the prescription that leads to the physical partition function as an effective change of the 
target space along the lines advocated in [21]. Let us also stress that, while our arguments were based on the $k=1$ theory, it is clear that a similar reasoning can be carried out for other levels.

The argument leading to $b$ being continuous also seems to exclude the smaller theories where part of the complex $\mathcal{J}$ is dropped, at least for general values of $k$. We shall see an exception in the case $k=-1 / 2$ later.

\section{$5 \quad$ Some selected applications}

This following section contains some selected applications of our general analysis. In the first subsection we shall compare our results with studies of the continuum limit of the integrable sl(2|1) $3-\overline{3}$ spin chain [9]. The agreement we find supports a new interpretation of lattice results. The second subsection is devoted to the $k=-1 / 2$ theory which was not included above, but we shall see that it shares many of the structures we uncovered throughout the last few sections.

\subsection{The $3-\overline{3}$ super-spin chain revisited.}

In [9] an integrable sl(2|1) invariant super-spin chain was studied using both analytical and numerical techniques. Its Hamiltonian acts on the tensor product $(3 \otimes \overline{3})^{\otimes L}$ where 3 and $\overline{3}$ stand for the representations $\{1 / 2\}_{ \pm}$in our previous terminology. It was argued that in the continuum limit this chain flows to a $\mathrm{SU}(2 \mid 1)$ WZNW model at level $k=1$. At the time, the WZNW model on the supergroup $\mathrm{SU}(2 \mid 1)$ had not been constructed and it seems instructive to revisit the issue now on the basis of our improved understanding of the continuum field theory. We shall see that the suggested identification with the continuum limit of the spin chain can be maintained, but some of the lattice results receive an interesting reinterpretation.

Let us begin by reviewing briefly some results on the spectrum of the lattice model. In [9] it was found analytically that this spectrum exhibits a unique ground state at $h=\bar{h}=$ 0 , which lies in the single "true singlet" of the model, i.e. it is a $\operatorname{sl}(2 \mid 1)$ invariant state that is not part of a larger indecomposable representation. This ground state corresponds to an extremely degenerate solution of the Bethe ansatz equations where all roots collapse to the origin. Besides the ground state, many excited states were also found. The lowest lying state above the ground state corresponds to a filled sea of some (non complex conjugate) 
string complexes. The rest of the spectrum is given by excitations obeying the usual pattern of holes and shifts of the sea. The scaled energies of these excitations over the ground state were found analytically to be

$$
\begin{aligned}
\frac{L}{2 \pi v} \Delta E=\frac{1}{4} & +\frac{1}{2}\left(\Delta N_{+}+\Delta N_{-}\right)^{2}+\frac{1}{8}\left(D_{+}+D_{-}\right)^{2}+ \\
& +C_{N}(L)\left(\Delta N_{+}-\Delta N_{-}\right)^{2}+C_{D}(L)\left(D_{+}-D_{-}\right)^{2} \\
\text { where } & C_{N}(L) \rightarrow 0, C_{D}(L) \rightarrow \infty \text { for } \quad L \rightarrow \infty
\end{aligned}
$$

Here, $\Delta N_{ \pm}, D_{ \pm}$are quantum numbers characterizing the Bethe ansatz solution. In the continuum limit, the quantity (5.1) is expected to converge to $x=h+\bar{h}$ (all weights in the $c=0$ theory), as usual. Formula (5.1) indicates an infinite degeneracy of the level $h=\bar{h}=1 / 8$ (obtained with $\Delta N_{+}=\Delta N_{-}=D_{+}=D_{-}=0$ say) in the limit $L \rightarrow \infty$. Numerical studies confirm this behavior: Indeed, they show that that an infinite number of levels converges to $h=\bar{h}=1 / 8$ as $L$ increases. This was already interpreted in [9] as indicating the existence of a continuum of conformal weights starting at $h=\bar{h}=1 / 8$ in the thermodynamic limit.

Although an analytical study of the asymptotic corrections to (5.1) seems still out of reach, numerical studies in a closely related model suggest that the leading contributions to $C_{N}$ and $C_{L}$ can be well fitted by the formulas

$$
C_{N}(L) \sim \frac{c}{\ln L}+\ldots \quad, \quad C_{D}(L) \sim \frac{4}{c} \ln L+\ldots
$$

for large number $2 L$ of lattice sites. When these leading terms are plugged back into the formula (5.1) for the spectrum of the lattice model, we see that the second line resembles very much the spectrum of a free boson which has been compactified to a circle with radius square of the order $\ln L$. In other words, if we assume that eqs. (5.2) are correct, the contribution from the "antisymmetric sector" (i.e. form excitations for which $\Delta N_{+}-\Delta N_{-}$or $D_{+}-D_{-}$are non zero) to the partition function can be estimated as

$$
\begin{aligned}
Z_{a n t i} & =\frac{1}{\eta \bar{\eta}} \sum_{e, m} q^{(e / R+m R / 2)^{2}} \bar{q}^{(e / R-m R / 2)^{2}} \\
& =\frac{R}{\sqrt{2}} \frac{1}{\sqrt{\operatorname{Im} \tau} \eta \bar{\eta}} \sum_{m, m^{\prime}} \exp \left(-\frac{\pi R^{2}\left|m \tau-m^{\prime}\right|^{2}}{2 \operatorname{Im} \tau}\right) \\
& \approx \frac{R}{\sqrt{2}} \frac{1}{\sqrt{\operatorname{Im} \tau} \eta \bar{\eta}}
\end{aligned}
$$


where $\eta(q)$ is Dedekind's eta function, as before. The divergence is proportional to $R$, i.e. to the size of the target space, as expected. We conclude that in the lattice model, the contribution from the antisymmetric sector to the partition function multiplies the contribution from the symmetric sector in eq. (5.1) by a term of the order of $\sqrt{\ln L}$. The ground state meanwhile, being a very degenerate Bethe ansatz solution, does not come with such an extra factor. The generating function of levels in the periodic sector will therefore have the form

$$
Z^{R}=1+\operatorname{cst} \sqrt{\ln L}\left[\frac{1}{\sqrt{\operatorname{Im} \tau} P \bar{P}}(q \bar{q})^{1 / 8}+\ldots\right]
$$

where the dots represent excitations from the symmetric sector in (5.1).

The first conclusion we draw is that the contribution of the continuum completely overrides the one from the discrete state (as would be the case in any quantum mechanics problem with discrete states and a continuum with delta function normalizable states), and that a properly normalized partition function does not see the singlet with $h=\bar{h}=0$. The resulting object is in good agreement with our conjectured partition function (4.17).

Reference [9] contained various failed attempts to build a conformal field theory containing both the continuum of representations $\{b, j=1 / 2\}$ and a single identity field associated with the representation $\{0\}$. Given our new insight into the continuum model, the problems to incorporate the singlet state may not come as a complete surprise. Although the free field construction at $k=1$ suggests the possibility of smaller theories, the study of modular invariants (as well as of four point functions, as we mentioned above) seems to preclude the appearance of the singlet representation on its own - i.e. without being part of a big indecomposable with vanishing super-dimension. In addition all the states we found in the continuum approach were non normalizable. Both observations lead us to speculate that eq. (4.17) represents the full operator content of the continuum limit, and that there is no discrete state associated with a true singlet. Put differently, the new investigation suggests that the true singlet observed on the lattice is an artifact of the regularization and does not belong to the continuum limit. Our new interpretation of the lattice results receives additional support from the very singular nature of the Bethe ansatz solution that corresponds to the singlet state. It would be interesting to check further the decoupling of the true singlet by studying the scaling behavior of matrix elements of lattice regularized current algebra generators. 
There is one more potential objection one might raise. Note that in our continuum theory fermionic and bosonic states are perfectly paired so that the Witten index of the $\mathrm{SU}(2 \mid 1)$ WZNW model is guaranteed to vanish. Meanwhile, for our lattice spin chain on the space $(3 \otimes \overline{3})^{\otimes L}$ one finds an excess by one for the number of bosonic states over the number of fermionic ones. Hence, the Witten index is non-zero on the lattice and one would naively expect the same to be true for the continuum limit, in conflict with what we have proposed above. In order to resolve this issue, we suggest that there exist different spin chains which give rise to the same continuum limit while possessing an excess of fermionic states over bosonic. More concretely, while we do not understand the whole structure yet, we have found 4 that the ground states of integrable chains of the type $(3 \otimes \overline{3})^{\otimes L} \otimes 3$ and $\overline{3} \otimes(3 \otimes \overline{3})^{\otimes L}$ scale to conformal weight $h=0$ as well (in fact the ground state energy is given exactly by $E_{0}=-$ length $\times e_{0}$ where $e_{0}$ has no finite size correction and is the same for all chains), but this time they come in the representation 3 (resp. $\overline{3}$ ). Once we sum over the various lattice models, the balance between bosonic and fermionic states may be restored even before taking the continuum limit.

\subsection{The WZNW model at $k=-1 / 2$}

Our investigation above was restricted to integer level $k$. But as we have mentioned before, these are some fractional values of $k$, in particular $k=-1 / 2$, which play an important role for applications. While we are not prepared to give a systematic account on fractional level theories, we would like to discuss briefly a model with $k=-1 / 2$. Our analysis will lead to the remarkable conclusion that the basic structure of this model is essentially the same as for integer $k$, only that there exist several components within the atypical sector, each of them being modeled after $\mathcal{J}$.

In this case $k=-1 / 2$, the relevant representation theory of the $\mathrm{sl}(2 \mid 1)$ current algebra is particularly simple. In fact, all relevant representations can be obtained from the vacuum sector $\{0\}^{\wedge}$ through application of spectral flow symmetries. It is not difficult to show that at $k=-1 / 2$ the automorphism $\gamma^{2}$ is inner, i.e. $\gamma^{2} \sim i d$. This means that application of $\gamma^{2}$ does not lead to any new representations. The remaining nontrivial automorphisms are of the form $\gamma_{+}^{n} \gamma^{\sigma}$ with $n \in \mathbb{Z}$ and $\sigma=0,1$. We shall denote the

\footnotetext{
${ }^{4}$ We thank F. Essler for kindly exploring this question numerically.
} 
corresponding irreducible representations of the $\mathrm{sl}(2 \mid 1)$ current algebra by

$$
\{(n, \sigma)\} \cong \gamma_{+}^{n} \gamma^{\sigma}\{0\}^{\wedge}
$$

By construction, this set closes under fusion. In fact, the fusion product simply amounts to a composition of the associated automorphism.

With the exception of the sectors labeled by $n=0, \pm 1$, the representations $\{(n, \sigma)\}$ do not contain a highest or lowest weight. The representation $\{(0,0)\}$ is to be identified with the vacuum representation. $\{(0,1)\}=\{0,1 / 2\}^{\wedge}$ is the only other admissible representation at $k=-1 / 2$. It is generated from the 4 -dimensional typical multiplet $\{0,1 / 2\}$ of ground states with conformal weight $h=1 / 2$. In addition, there are four more highest/lowest weight weight representations which are erected over the atypical discrete series representations $\left\{\left(-1, \sigma_{ \pm}\right)\right\}=\{(-,-1 / 4)\}_{ \pm}^{\wedge}$ and $\left\{\left(1, \sigma_{ \pm}\right\}=\{(+,-1 / 4)\}_{ \pm}^{\wedge}\right.$ corresponding to a negative spin $j=-1 / 4$. The choice of the sign in the first argument of the bracket determines on whether the representation is highest $(-)$ or lowest $(+)$ weight. The subscript, on the other hand corresponds to the two different choices of the parameter $b$ that make these representations atypical. All four representations possess ground states of conformal weight $h=0$. In all other representations $\{(n, \sigma)\}$ with $|n| \geq 2$, the conformal weight is unbounded from below.

Since we can generate every representations from $\{0\}^{\wedge}$, is suffices to display the character of the vacuum representation,

$$
\chi_{\{0\}}(q, z, \xi)=\frac{1}{2}\left[\frac{\vartheta_{3}\left(q, \xi^{1 / 2}\right)}{\vartheta_{4}\left(q, z^{1 / 2}\right)}+\frac{\vartheta_{4}\left(q, \xi^{1 / 2}\right)}{\vartheta_{3}\left(q, z^{1 / 2}\right)}\right] .
$$

We shall explain the origin of this formula in a moment. Characters of all the other representations are obtained from the vacuum character $\chi_{\{0\}}$ through

$$
\chi_{\{(n, \sigma)\}}(q, z, \xi)=\gamma_{+}^{n} \gamma^{\sigma} \chi_{\{0\}}(q, z, \xi)=\xi^{-\frac{n}{4}-\frac{\sigma}{2}} z^{-\frac{n}{4}} q^{\frac{n+1}{2} \sigma} \chi_{\{0\}}\left(q, q^{-n-2 \sigma} \xi, q^{n z} z\right) .
$$

To derive the above character formula and for the subsequent discussion we note that the $\operatorname{sl}(2 \mid 1)$ current algebra at level $k=-1 / 2$ possesses a free field representation which employs the same free fields as in the case of the $k=1$ theory, i.e. two free bosonic fields $\phi$ and $\phi^{\prime}$ with space-like and time-like signature, respectively, and a pair of symplectic fermions $\eta_{1}, \eta_{2}$. The bosonic sl $(2 \mid 1)$ currents read

$$
\begin{aligned}
& E^{+}(z)=\frac{1}{2} e^{-2 i \varphi^{\prime}(z)} \partial^{2} \eta_{1}(z) \partial \eta_{1}(z) \quad, \quad H(z)=\frac{i}{2} \partial \phi^{\prime}(z) \\
& E^{-}(z)=\frac{1}{2} e^{2 i \varphi^{\prime}(z)} \partial^{2} \eta_{2}(z) \partial \eta_{2}(z) \quad, \quad B(z)=\frac{i}{2} \partial \phi(z)
\end{aligned}
$$


Note that, unlike in the case of $k=1$, the bosonic currents involve the symplectic fermions and the time-like free boson. For the fermionic currents one finds

$$
\begin{aligned}
& V^{-}(z)=\frac{1}{\sqrt{2}} e^{-i\left(\varphi(z)-\varphi^{\prime}(z)\right)} \partial \eta_{2}(z) \quad, \quad V^{+}(z)=\frac{1}{\sqrt{2}} e^{-i\left(\varphi(z)+\varphi^{\prime}(z)\right)} \partial \eta_{1}(z) \\
& W^{-}(z)=\frac{1}{\sqrt{2}} e^{i\left(\varphi(z)+\varphi^{\prime}(z)\right)} \partial \eta_{2}(z) \quad, \quad W^{+}(z)=\frac{1}{\sqrt{2}} e^{i\left(\varphi(z)-\varphi^{\prime}(z)\right)} \partial \eta_{1}(z)
\end{aligned}
$$

As in the case of the $k=1$ theory, the free field construction determines a consistent model with a $\operatorname{sl}(2 \mid 1)$ current algebra symmetry. If we do not include the symplectic fermions (note that once more the currents only involve derivatives), but only their derivatives then the state space reads

$$
\mathcal{H}_{k=-1 / 2}=\bigoplus_{n, \sigma}\{(n, \sigma)\}^{\wedge} \times\{(-n, \sigma)\}^{\wedge}
$$

Since the spectral flow automorphisms $\gamma_{ \pm}$and $\gamma$ correspond to multiplication with the fields

$$
\gamma_{ \pm} \leftrightarrow e^{-\frac{i}{2}\left(\phi^{\prime} \pm \phi\right)} \quad, \quad \gamma \leftrightarrow e^{-i \phi}
$$

is is fairly easy to write down at least one field in each sector of the model,

$$
\{(n, \sigma)\}^{\wedge} \times\{(-n, \sigma)\}^{\wedge} \text { contains } \quad e^{-i \frac{n}{2} \phi^{\prime}-i \frac{n+2 \sigma}{2} \phi}
$$

The space $\mathcal{H}_{k=-1 / 2}$ contains $\mathrm{R}$ sector representations only, but it is certainly possible to include the NS sector by adding the image under the spectral flow $\vartheta$. Since this works just in the same way as above, we shall not repeat the discussion here.

Even though all the representations we are working with are atypical, the state space decomposes into irreducible building blocks. This is quite different from the structure of the atypical sectors $\mathcal{J}^{\wedge}$ we described above. On the other hand, is is very similar to one of the consistent theories with $k=1$ that we described at the end of section 4.2. In the $k=1$ theory, the singular vectors of the indecomposable block $\mathcal{J}^{\wedge}$ were decoupled by restricting to a theory that contained only derivatives of the fermionic fields. Conversely, the experience from $k=1$ suggest that in the $k=-1 / 2$ case we may be able to construct a theory with a more complicated atypical sector by including one or both of the symplectic fermion fields $\eta_{1}$ and $\eta_{2}$ [22].

We claim that in case we include both fermionic zero modes we end up with an atypical structure that decomposes into four different blocks, each of them being built in the same 
way as our sector $\mathcal{J}^{\wedge}$. We shall present the analysis only for the block that contains the vacuum sector $\{0\}^{\wedge}$. The other three sectors are obtained by acting with $\gamma, \gamma_{+}$and $\gamma_{+} \circ \gamma$. Let us start our discussion with the field $\eta_{1} \eta_{2}$. Any action with $V^{ \pm}, W^{ \pm}$and $E^{ \pm}$will remove one of the two fermionic zero modes and hence $\eta_{1} \eta_{2}$ sits at the top of a sector $\{0\}^{\wedge}$. The action of $V^{+}, W^{+}$and $E^{+}$takes us from here into a set of fields which all contain a factor $\eta_{1}$. These fields can be shown to belong to a sector that is isomorphic to $\{(2,0)\} \cong \gamma_{+}^{2}\{0\}^{\wedge}$. Further application of $V^{-}, W^{-}$and $E^{-}$bring us to a set of fields that contain only derivatives of fermions. These form a subrepresentation $\{0\}^{\wedge}$ at the bottom of our atypical representation. A similar analysis applies if we act with $V^{-}, W^{-}$and $E^{-}$

first. This time, we descend to $\{0\}^{\wedge}$ via the sector $\{(-2,0)\} \cong \gamma_{+}^{-2}\{0\}^{\wedge}$. Continuing along this line of thoughts, one can see that the sectors $\{(2 n, 0)\}, n \in \mathbb{Z}$, form the composition series for an indecomposable representation $\mathcal{J}^{\wedge}$ with $\{(2 n, 0)\}$ in place of $\{n / 2\}$. The state space of the maximal theory therefore decomposes into four indecomposable blocks. Once more, there are two intermediate theories, each of which has four saw-blade shaped atypical sectors. They are obtained if we omit either $\eta_{1}$ or $\eta_{2}$ (but not their derivatives, of course) from the above maximal theory.

Even though we are not prepared to analyze WZNW models for generic fractional levels, it is remarkable that the structure we have first uncovered in our minisuperspace limit, re-appears even for $k=-1 / 2$. It seems very likely that the same is true for a generic choice of the level.

\section{Conclusions and Outlook}

An obvious conclusion of our study is that WZNW models on supergroups are interesting examples of logarithmic CFTs, much richer than it has been anticipated in earlier works. Gurarie (see [23] and references therein), for instance, argued that super WZNW models with $c=0$ could be considered as made of two "decoupled" component theories with opposite values of the central charge, an observation justified in part by the fact that in the GL(1|1) WZNW model, the stress energy tensor belongs naturally to a four dimensional GL(1|1) multiplet in which $L_{0}$ is diagonalizable, and hence $T$ has no "non trivial logarithmic partner". The SU(2|1) WZNW clearly does not obey any such decoupling. In fact, restricting to the right moving current algebra as in [23] we see that the identity field belongs to a projective representation of the zero mode algebra on which the Casimir 
- and hence $L_{0}$ - is not diagonalizable. Applying $L_{-2}$ to this representation produces a Virasoro Jordan cell at level $h=2$ and a non trivial logarithmic partner of the stress energy tensor. This can be seen quite explicitely in the case $k=1$ where, within the free field representation (and similarly to the case of symplectic fermions), the field

$$
t(z):=: \eta_{1}(z) \eta_{2}(z) T(z):
$$

is a logarithmic partner of

$$
T:=: \partial \eta_{1}(z) \partial \eta_{2}(z):-\frac{1}{2}:(\partial \phi(z))^{2}:+\frac{1}{2}:\left(\partial \phi^{\prime}(z)\right)^{2}:
$$

Note that the whole structure of indecomposables is in fact much more complicated than envisioned in [23] when the interplay of left and right current algebras is taken into account.

Even though the structure of the state space is rather difficult when analyzed with respect to the combined left and right action, it is surprisingly simple once we restrict to either the left or the right action alone. Note that the Lie superalgebra sl(2|1) has a large number of indecomposables (see e.g. [11]) from which only a very distinguished sub-class does actually occur within the state space of our model. In fact, we have seen above that all states (both in the minisuperspace theory and the full field theory) transform according to the so-called projective representations of $\mathrm{sl}(2 \mid 1)$, i.e. either in typicals and projective covers of atypicals. This is not to say, however, that other representations of $\operatorname{sl}(2 \mid 1)$ have no relevance for sigma models on supergroups. In addition to the left and right regular representation there is yet one more important symmetry that arises from the adjoint action of $\operatorname{sl}(2 \mid 1)$ on the state space. With respect to the latter, states can transform in other indecomposables. The underlying mathematical structure turns out to be quite intriguing and will be described elsewhere. Since the adjoint action is left unbroken by maximally symmetric boundary conditions, the resulting decomposition should have applications, in particular to the study of boundary conditions for sigma models on supergroups.

As a final comment let us point out one generic feature we have encountered in both GL(1|1) and $\mathrm{SU}(2 \mid 1)$, namely that the contribution of the indecomposable sector $\mathcal{J}$ simply makes up for the subtractions in the atypical sectors of the theory, so that the partition function sees only contributions from Kac modules, and has a simple factorized form. This behavior is sufficient for a modular invariant partition function but it is not necessary. 
The potential existence of different versions of the theory where only parts of the complex $\mathcal{J}$ appear, requires more study. We note that some hints in this direction are provided by the study of four point functions. In the case $k=1$, the four point function of the fields in the $\{0,1 / 2\}$ representation has been studied in detail. It turns out that the KZ equations factorize, and that it is possible to decouple one conformal block. Two blocks remain, leading to logarithmic dependence, and indicating that the identity field remains part of an indecomposable representation. This suggests that the smallest theory, where the complex $\mathcal{J}$ is reduced to an infinite sum of irreducibles, cannot appear in the $\operatorname{sl}(2 \mid 1)$ WZNW model. In the case $k=-1 / 2$ meanwhile it is possible to decouple two conformal blocks, leaving only the identity field, and indicating that the smallest theory does make sense this time - a feature consistent with the free field representation and the modular invariant. The $\operatorname{sl}(2 \mid 1)$ WZNW model at fractional level and the explicit construction of consistent theories with a truncated atypical sector certainly deserve a more systematic investigation.

Acknowledgments: We thank Fabian Essler, Gerhard Götz, Thomas Quella and Anne Taormina for interesting conversations. V.S. would like to thank the SPhT for the warm hospitality during several stays. This work was partially supported by the EU Research Training Network grants "Euclid", contract number HPRN-CT-2002-00325 and "ForcesUniverse", contract number MRTN-CT-2004-005104.

\section{Appendix A: The right regular representation}

In this appendix we would like to prove the decomposition formula for the right regular representation. We shall use the same notations that were introduced in section 2.2. In order to analyze the decomposition of the space of functions under the right regular action of $\operatorname{sl}(2 \mid 1)$, we shall first study its restriction to the Lie sub-superalgebra gl(1|1). More precisely, we shall make use of the following embedding

$$
\epsilon\left(\psi^{+}\right)=F^{+}, \epsilon\left(\psi^{-}\right)=\bar{F}^{-}, \epsilon(E)=B-H, \epsilon(N)=B+H .
$$

The main technical Lemma of this section implies that under the action of $g l(1 \mid 1)$, the space $\mathcal{H}$ of functions on the supergroup $\mathrm{SU}(2 \mid 1)$ decomposes into projectives only. 
Lemma: Under the action of $R_{X} \equiv R_{\epsilon(X)}$ of the generators $X \in$ gl(1|1), the space $\mathcal{H}$ of functions of $S U(2 \mid 1)$ decomposes according to

$$
\mathcal{H} \cong \bigoplus_{j} \bigoplus_{b=-j}^{j} \mathcal{P}(2 b+1) \oplus 2 \cdot \mathcal{P}(2 b) \oplus \mathcal{P}(2 b-1) \oplus \mathcal{T}
$$

Here, $\mathcal{T}$ is a direct sum of typical gl(1|1) representations and $\mathcal{P}(a)$ denotes the projective cover of the atypical irreducible $\langle a\rangle$.

Before we prove this statement, let us formulate two consequences for the right regular representation of $\operatorname{sl}(2 \mid 1)$. To begin with, let us recall from [11] that an $\mathrm{sl}(2 \mid 1)$ representation $\pi$ descends on a projective representation of the embedded gl(1|1) algebra if and only if $\pi$ is projective. Our lemma claims that the $\mathrm{gl}(1 \mid 1)$ action on $\mathrm{H}$ contains only projectives. Hence, the same must be true for the right regular action of $\operatorname{sl}(2 \mid 1)$.

Proof of Lemma: For the proof it will be useful to introduce the following odd functions

$$
\bar{\theta}_{ \pm}:=e^{\mp i z / 2} D_{( \pm 1 / 2) \nu}^{1 / 2}\left(g^{-1}\right) \bar{\eta}_{\nu} \quad, \quad \tilde{\eta}_{-}=e^{i z} \eta_{-}
$$

It is not difficult to see that the space $\mathcal{H}$ is spanned by functions of the form

$$
F_{a b}^{n, j} \Lambda=e^{i n z} D_{a b}^{j}(g) \Lambda\left(\tilde{\eta}_{-}, \eta_{+}, \bar{\theta}_{ \pm}\right)
$$

where $\Lambda\left(\tilde{\eta}_{-}, \eta_{+}, \bar{\theta}_{ \pm}\right)$is an arbitrary element in the algebra generated by the arguments. It is very easy to describe explicitly the space of functions which are organized in atypicals of $g l(1 \mid 1)$. The latter is characterized by the vanishing of $R_{E}$,

$$
\mathcal{A}_{R}=\left\{\Phi \in \mathcal{H} \mid\left(i \partial_{z}+R_{h}^{0}+\eta_{-} \partial_{-}\right) \Phi=0\right\}
$$

We can easily solve the equation for $\Phi$ and describe the space $\mathcal{A}_{R}$ explicitly. In fact, it is spanned by the functions

$$
F_{a b}^{b, j} \Lambda=e^{i b z} D_{a b}^{j}(g) \Lambda\left(\tilde{\eta}_{-}, \eta_{+}, \bar{\theta}_{ \pm}\right)
$$

On the subspace $\mathcal{A}^{R}$ the other generators of gl(1|1) simplify to

$$
\begin{gathered}
R_{N} \Phi=\left(-2 i \partial_{z}-\eta_{-} \partial_{-}-\eta_{+} \partial_{+}\right) \Phi \\
R_{\Psi^{+}} \Phi=-i \partial_{+} \Phi \quad, \quad R_{\Psi^{-}} \Phi=i e^{-i z / 2} D_{\nu(-1 / 2)}^{1 / 2}(g) \bar{\partial}_{-\nu}-i \eta_{-} R_{E^{-}}^{0}
\end{gathered}
$$


for all $\Phi \in \mathcal{A}_{R}$. The representation of gl(1|1) can be restricted to the space $\mathcal{A}_{R}^{\prime}$ of all elements $\Phi \in \mathcal{A}_{R}$ such that $\eta_{-} \partial_{-} \Phi=\Phi$. A short look on the action of the gl(1|1) generators reveals that

$$
\mathcal{A}_{R}^{\prime}=\bigoplus_{j} \bigoplus_{b=-j}^{j} \mathcal{P}(2 b+1) \oplus \mathcal{P}(2 b)
$$

Similarly, we see that

$$
\mathcal{A}_{R} / \mathcal{A}_{R}^{\prime}=\bigoplus_{j} \bigoplus_{b=-j}^{j} \mathcal{P}(2 b) \oplus \mathcal{P}(2 b-1)
$$

Since all representations are projective we conclude that

$$
\mathcal{A}_{R}=\bigoplus_{j} \bigoplus_{b=-j}^{j} \mathcal{P}(2 b+1) \oplus 2 \cdot \mathcal{P}(2 b) \oplus \mathcal{P}(2 b-1)
$$

This concludes the proof of our Lemma.

\section{References}

[1] D. Bernard, (Perturbed) conformal field theory applied to 2D disordered systems: An introduction, hep-th/9509137.

[2] M. J. Bhaseen, J. S. Caux, I. I. Kogan and A. M. Tsvelik, Disordered dirac

fermions: the marriage of three different approaches, Nucl. Phys. B618 (2001) 465-499 [cond-mat/0012240].

[3] Z. Maassarani and D. Serban, Non-unitary conformal field theory and logarithmic operators for disordered systems, Nucl. Phys. B489 (1997) 603-625 hep-th/9605062.

[4] A. W. W. Ludwig, A free field representation of the osp $(2 \mid 2)$ current algebra at level $k=-2$, and Dirac fermions in a random $S U(2)$ gauge potential, cond-mat/0012189.

[5] P. Bowcock, M. Hayes and A. Taormina, Characters of admissible representations of the affine superalgebra sl(2|1), Nucl. Phys. B510 (1998) 739-764 hep-th/9705234. 
[6] L. Rozansky and H. Saleur, Quantum field theory for the multivariable Alexander-Conway polynomial, Nucl. Phys. B376 (1992) 461-509.

[7] V. Schomerus and H. Saleur, The GL(1|1) WZW model: From supergeometry to logarithmic CFT, hep-th/0510032.

[8] G. Gotz, T. Quella and V. Schomerus, The WZNW model on PSU(1,1|2), hep-th/0610070.

[9] F. H. L. Essler, H. Frahm and H. Saleur, Continuum limit of the integrable sl(2|1) $3-\overline{3}$ superspin chain, Nucl. Phys. B712 (2005) 513-572 [cond-mat/0501197.

[10] L. Frappat, P. Sorba and A. Sciarrino, Dictionary on Lie algebras and superalgebras. Academic Press Inc., San Diego, CA, 2000. Extended and corrected version of the E-print hep-th/9607161].

[11] G. Götz, T. Quella and V. Schomerus, Representation theory of sl(2|1), hep-th/0504234.

[12] V. G. Kac, Lie superalgebras, Adv. Math. 26 (1977) 8-96.

[13] A. Hüffmann, On representations of supercoalgebras, J. Phys. A27 (1994) 6421-6432 [hep-th/9403100].

[14] P. Bowcock and A. Taormina, Representation theory of the affine lie superalgebra sl $(2 \mid 1, C)$ at fractional level, Commun. Math. Phys. 185 (1997) 467-493 hep-th/9605220].

[15] M. Hayes and A. Taormina, Admissible sl $(2 \mid 1, C)(k)$ characters and parafermions, Nucl. Phys. B529 (1998) 588-610 hep-th/9803022.

[16] A. M. Semikhatov and A. Taormina, Twists and singular vectors in $\widehat{s l}(2 \mid 1)$ representations, Theor. Math. Phys. 128 (2001) 1236-1251 hep-th/0311166].

[17] A. M. Semikhatov, A. Taormina and I. Y. Tipunin, Higher level Appell functions, modular transformations, and characters, Comm. Math. Phys. 255 (2005) 469-512 math.qa/0311314. 
[18] L. Rozansky and H. Saleur, $S$ and T matrices for the $U(1 \mid 1)$ WZW model: Application to surgery and three manifolds invariants based on the Alexander-Conway polynomial, Nucl. Phys. B389 (1993) 365-423 hep-th/9203069.

[19] L. J. Mordell, Acta Math. 61 (1933) 323.

[20] T. Apostol, Modular Functions and Dirichlet Series in Number Theory, vol. 41. Springer-Verlag, Berlin, Heidelberg, New York, 1997.

[21] M. Bocquet, D. Serban and M. R. Zirnbauer, Disordered 2d quasiparticles in class d: Dirac fermions with random mass, and dirty superconductors, Nuclear Physics B 578 (2000) 628.

[22] F. Lesage, P. Mathieu, J. Rasmussen and H. Saleur, Nucl. Phys. B686 (2004) 313.

[23] V. Gurarie and A. W. W. Ludwig, Conformal field theory at central charge $c=0$ and two-dimensional critical systems with quenched disorder, hep-th/0409105. 